\title{
Existence and asymptotic behavior of Radon measure-valued solutions for a class of nonlinear parabolic equations
}

\author{
Quincy Stévène Nkombo ${ }^{1,2 *}$, Fengquan Li ${ }^{1}$ and Christian Tathy ${ }^{2}$
}

"Correspondence:
quincysnk@yahoo.fr
'School of Mathematical Sciences,
Dalian University of Technology,
Dalian 116024, China
2Laboratoire de Mécanique,
Energétique et Ingénierie Ecole
Nationale Supérieure
Polytechnique, Université Marien
Ngouabi, BP 69, Brazzaville, Republic
of Congo

"Correspondence: quincysnk@yahoo.fr Dalian 116024, Chin Laboratoire de Mécanique, Nationale Supérieure

\section{Springer}

\begin{abstract} bounded Radon measure. This problem is described as follows:

$$
\begin{cases}u_{t}=\alpha u_{x x}+\beta[\varphi(u)]_{x x}+f(u) & \text { in } Q:=\Omega \times(0, T), \\ u=0 & \text { on } \partial \Omega \times(0, T), \\ u(x, 0)=u_{0}(x) & \text { in } \Omega,\end{cases}
$$
\end{abstract}

In this paper we address the weak Radon measure-valued solutions associated with the Young measure for a class of nonlinear parabolic equations with initial data as a

where $T>0, \Omega \subset \mathbb{R}$ is a bounded interval, $u_{0}$ is nonnegative bounded Radon measure on $\Omega$, and $\alpha, \beta \geq 0$, under suitable assumptions on $\varphi$ and $f$. In this work we prove the existence and the decay estimate of suitably defined Radon measure-valued solutions for the problem mentioned above. In particular, we study the asymptotic behavior of these Radon measure-valued solutions.

MSC: 35K65; 35K55; 35B40; 35R06; 28A50; 28A33

Keywords: Radon measure-valued solution; Nonlinear parabolic equations; Young measure; Asymptotic behavior

\section{Introduction}

In this paper we address the existence, decay estimate, and the asymptotic behavior of solutions for the following problem:

$$
\begin{cases}u_{t}=[\psi(u)]_{x x}+f(u) & \text { in } Q:=\Omega \times(0, T), \\ u=0 & \text { on } \partial \Omega \times(0, T), \\ u(x, 0)=u_{0}(x) & \text { in } \Omega,\end{cases}
$$

where $T>0, \Omega \subset \mathbb{R}$ is a bounded interval, $u_{0}$ is nonnegative bounded Radon measure on $\Omega$ under suitable assumptions on $\psi$ and $f$ expressed as follows:

$$
\psi(s)=\alpha s+\beta \varphi(s)
$$

(c) The Author(s) 2021. This article is licensed under a Creative Commons Attribution 4.0 International License, which permits use, sharing, adaptation, distribution and reproduction in any medium or format, as long as you give appropriate credit to the original author(s) and the source, provide a link to the Creative Commons licence, and indicate if changes were made. The images or other third party material in this article are included in the article's Creative Commons licence, unless indicated otherwise in a credit line to the material. If material is not included in the article's Creative Commons licence and your intended use is not permitted by statutory regulation or exceeds the permitted use, you will need to obtain permission directly from the copyright holder. To view a copy of this licence, visit http://creativecommons.org/licenses/by/4.0/ 
for any $(\alpha, \beta) \in \mathbb{R}_{+} \times \mathbb{R}_{+}$, where the function $\varphi$ satisfies the following assumptions:

$$
\left\{\begin{array}{l}
\text { (i) } \varphi \in L^{\infty}\left(\mathbb{R}_{+}\right) \cap C^{2}\left(\mathbb{R}_{+}\right), \quad \varphi(0)=0, \quad \varphi^{\prime}>0 \quad \text { in } \mathbb{R}_{+} \\
\text {(ii) } \varphi^{\prime}, \varphi^{\prime \prime} \in L^{\infty}\left(\mathbb{R}_{+}\right) \\
\text {(iii) } \varphi(s) \rightarrow \gamma \quad \text { as } s \rightarrow+\infty \\
\text { (iv) } \frac{\left|\varphi^{\prime \prime}\right|}{\varphi^{\prime}} \leq m_{0} \quad \text { in } \mathbb{R}_{+}^{*} \text { for some } m_{0} \in \mathbb{R}_{+}^{*}
\end{array}\right.
$$

where $\mathbb{R}_{+} \equiv[0,+\infty), \mathbb{R}_{+}^{*} \equiv(0,+\infty)$, and $\gamma \in(0,+\infty)$. A typical example of the function $\varphi$ is given by

$$
\varphi(s)=\gamma\left[1-e^{1-(1+s)^{m}}\right] \text { and } \varphi(s)=\int_{0}^{s} e^{-(1+z)^{m}} d z,
$$

where $0<m \leq 1, s \geq 0$.

The function $f$ verifies the following assumption:

$$
\left\{\begin{array}{l}
\text { (i) } f \in L^{\infty}\left(\mathbb{R}_{+}\right) \cap C^{1}\left(\mathbb{R}_{+}\right), \quad f(0)=0, \\
\text { (ii) } f^{\prime} \in L^{\infty}\left(\mathbb{R}_{+}\right) \text {and } f^{\prime}>0 \quad \text { in } \mathbb{R}_{+} .
\end{array}\right.
$$

The example of the function $f$ is

$$
f(s)=C\left[1-\frac{1}{(1+s)^{m}}\right] \text { and } f(s)=\int_{0}^{s} e^{-z^{m}} d z .
$$

The assumption of the function $\psi$ in (1.1) and hypothesis $(\mathrm{H})$ are summarized as follows:

$$
\left\{\begin{array}{l}
\text { (i) } \psi, \psi^{\prime}, \psi^{\prime \prime} \in C\left(\mathbb{R}_{+}\right), \quad \psi(0)=0, \quad \psi^{\prime}>0 \quad \text { in } \mathbb{R}_{+} \\
\text {(ii) } \psi^{\prime}, \psi^{\prime \prime} \in L^{\infty}\left(\mathbb{R}_{+}\right), \\
\text {(iii) } \frac{\left|\psi^{\prime \prime}\right|}{\psi^{\prime}} \leq m_{0} \quad \text { in } \mathbb{R}_{+}^{*}, \quad \text { for some } m_{0} \in \mathbb{R}_{+}^{*} \\
\text { (iv) } \lim _{s \rightarrow+\infty} \frac{\psi(s)}{s}=\alpha \in \mathbb{R}_{+}
\end{array}\right.
$$

where $\psi^{\prime}$ and $\psi^{\prime \prime}$ are the first derivative and the second derivative of the function $\psi$ respectively. Similarly, hypothesis $(\mathrm{G})$ is inferred from assumption $(\mathrm{H})$ and the expression of the function $\psi$ given by (1.1). Since $\psi(s) \rightarrow+\infty$ as $s \rightarrow+\infty$, problem (P) is not degenerate at infinity. However, problem $(\mathrm{P})$ is degenerate at zero.

Remark 1.1 By assumption (R) and the mean-value theorem, it is worthy observing that

$$
\lim _{s \rightarrow+\infty} \frac{f(s)}{s}=f^{\prime}(+\infty)
$$

Then, for every $\epsilon>0$, there exists $M=M(\epsilon)>0$ such that

$$
-\epsilon s+f^{\prime}(+\infty) s \leq f(s) \leq f^{\prime}(+\infty) s+\epsilon s \quad \text { if } s \geq M
$$

By the example of the function $f$ in (1.2)-(1.3), it implies $f^{\prime}(+\infty)=0$. We notice that the function $f$ can also satisfy assumption (R) with $f^{\prime}(+\infty) \neq 0$. 
Throughout this paper, we consider the solutions of problem (P) as maps from $(0, T)$ to the cone of nonnegative bounded Radon measure on $\Omega$, which verify $(\mathrm{P})$ in the following sense: for a suitable class of test function $\xi$, there holds

$$
\begin{aligned}
& \int_{Q}\left\{u_{r} \xi_{t}+\psi^{*} \xi_{x x}\right\} d x d t+\int_{0}^{T}\left\langle u_{s}(\cdot, t), \bar{\xi}_{v}(\cdot, t)\right\rangle_{\Omega} d t \\
& \quad+f^{\prime}(+\infty) \int_{0}^{T}\left\langle u_{s}(\cdot, t), \xi(\cdot, t)\right\rangle_{\Omega} d t+\int_{Q} f^{*} \xi d x d t=\left\langle u_{0}, \xi(\cdot, 0)\right\rangle_{\Omega}
\end{aligned}
$$

where $\bar{\xi}_{v}=\xi_{t}+\alpha \xi_{x x}$ (see Definition 2.1). Here the measure $u(\cdot, t)$ is defined for a.e. $t \in$ $(0, T), u_{r} \in L^{1}(Q)$.

The one-dimensional degenerate parabolic equations with initial data have been intensively investigated in several decades. Similarly, the nonlinear parabolic equation (P) has been studied by many authors such as (see $[2-4,6,32])$. Within the type of problem (P), we consider the problem studied in [2]:

$$
\begin{cases}u_{t}=[\widetilde{\phi}(u(x, t))]_{x x}+h(u(x, t)) & x \in(-L, L), t>0, \\ u(-L, t)=u(L, t)=0, & x \in(-L, L), \\ u(x, 0)=u_{0}(x) \geq 0 & \end{cases}
$$

where $L>0$ and the functions $\widetilde{\phi}$ and $h$ verify the suitable assumptions (see [2] for more details). In [2], the authors studied the existence, uniqueness, and regularity of the solutions to problem (A.1) when $u_{0} \in L^{\infty}(-L, L)$. Meanwhile, the main purpose of the study of problem (A.1) is the convergence of the solutions when $t \rightarrow+\infty$. The difference between problem (A.1), the references $[3,4,6,32]$, and problem $(\mathrm{P})$ is the assumptions which satisfy the functions $\psi, f$ and the initial data $u_{0}$.

In the literature of one-dimensional nonlinear parabolic equations with initial data, there are many studies of the kind of problem $(\mathrm{P})$ without the source term $(f(u)=0)$ (for instance, see $[5,11,28,29,31,33-40])$. In [28], the authors studied the following problem:

$$
\begin{cases}u_{t}=[\phi(u)]_{x x} & \text { in } Q:=\Omega \times(0, T) \\ \phi(u)=0 & \text { on } \partial \Omega \times(0, T) \\ u(x, 0)=u_{0}(x) & \text { in } \Omega\end{cases}
$$

where $T>0, \Omega \subset \mathbb{R}$ is a bounded interval, $u_{0}$ is nonnegative bounded Radon measure on $\Omega$, and the function $\phi$ is nonmonotone and satisfies the hypothesis including $(\mathrm{H})$ (see [28] for more details).

In [28], the authors dealt with the existence of the weak Radon measure-valued solutions associated with the Young measure. The difference between problem (A.2) [5, 11, 29, 31, 33-40] and problem ( $\mathrm{P}$ ) remains the hypothesis on the function $f$ given by $(\mathrm{R})$.

In [14], the authors addressed the existence, uniqueness, and the qualitative properties of the Radon measure-valued solutions associated with the Young measure to the first order scalar conservation laws with Radon measure as initial data. The problem studied 
in $[14]$ is

$$
\begin{cases}u_{t}+[g(u)]_{x}=0 & \text { in } \mathbb{R} \times(0, T) \\ u(x, 0)=u_{0}(x) & \text { in } \mathbb{R}\end{cases}
$$

where $u_{0}$ is nonnegative bounded Radon measure on $\Omega$ and the function $g$ verifies hypothesis $(\mathrm{G})$.

Before we study the general problem of (P), we need to point out the particular cases of such a problem and their results. For instance $\alpha=0$, problem $(\mathrm{P})$ is nonlinear degenerate parabolic equations, and this kind of nonlinear degenerate parabolic equations is similar to

$$
\begin{cases}u_{t}=\operatorname{div}(A(x, t, u) \nabla u)+F(x, t, u) & \text { in } Q:=\Omega \times(0, T), \\ u=0 & \text { on } \partial \Omega \times(0, T), \\ u(x, 0)=u_{0}(x) & \text { in } \Omega,\end{cases}
$$

where $T>0, \Omega \subset \mathbb{R}^{N}(N \geq 2)$ is an open bounded domain with smooth boundary $\partial \Omega$ and $u_{0}$ is a finite Radon measure on $\Omega$. The operator $A(x, t, s)$ is weakly coercive and diffuse and $F(x, t, u)$ verifies the suitable hypothesis (see [1] for more details). In [1], the authors proved the existence and qualitative properties of the Radon measure-valued solutions associated with the Young measure. Another difference between problem (A.4) and (P) is the assumption which fulfills the function $F(x, t, u)$. Indeed, the hypothesis of the function $F(x, t, u)$ is different from assumption $(\mathrm{R})(f(u)$ verifies hypothesis $(\mathrm{R})$ of problem $(\mathrm{P}))$. On the other hand, the problems studied in the papers [44-46] are closely formulated as in (A.4), where the expression for the source term $F(x, t, u)$ is more regular and the diffusionterm $A(x, t, s)$ takes part on the modeling of real phenomena from mathematical biology and physics. Furthermore, the authors in [44-46] dealt with the properties of weak and classical solutions.

Assuming that $\beta=0$, problem $(\mathrm{P})$ is reduced to the semilinear heat equation with Radon measure as initial data described as follows:

$$
\begin{cases}u_{t}=u_{x x}+f(u) & \text { in } Q:=\Omega \times(0, T) \\ u=0 & \text { on } \partial \Omega \times(0, T) \\ u(x, 0)=u_{0}(x) & \text { in } \Omega\end{cases}
$$

where $\alpha \equiv 1, T>0, \Omega \subset \mathbb{R}$ is a bounded interval, $u_{0}$ is nonnegative bounded Radon measure on $\Omega$. By [19, 20], problem (A.5) admits unique weak solutions which are not Radon measure-valued associated with the Young measure. However, in [17] the authors showed the existence, qualitative properties, and decay estimate of the Radon measure-valued solutions to the Cauchy problem of (A.5). Throughout this paper, we consider the case $\alpha>0$ and $\beta>0$, and we notice that the result of this paper is not true for $\alpha=0$.

The goal of this paper is threefold. Firstly, we study the existence of the Radon measurevalued solutions associated with the Young measure introduced in [1] and the other technical tools stated in $[14,28]$. 
Secondly, we establish the decay estimate of the Radon measure-valued solutions to problem (P). We note that the proof of the existence of the Radon measure-valued solutions and the decay estimate of these weak solutions focus on the natural approximation method.

Thirdly, we analyze the asymptotic behavior of the Radon measure-valued solutions. To this purpose, we construct the pseudo-stationary solutions which are Radon measurevalued solutions to the nonlinear elliptic equations. Then the result of the asymptotic behavior of solutions follows from the use of the natural approximation method.

The novelty of this paper is twofold. Firstly, we study the decay estimate of the Radon measure-valued solutions of a class of nonlinear parabolic equations. Finally, we study the asymptotic behavior of these Radon measure-valued solutions.

The plan of this paper is organized as follows. In the next section, we recall some preliminaries about Radon measures and Young measures. Then, in Sect. 3, we state the main results, while in Sects. $4-7$ we prove the main results.

\section{Preliminaries}

\subsection{Radon measures}

Let $\mathcal{M}(\Omega)$ be the space of bounded Radon measures on $\Omega$, and $\mathcal{M}^{+}(\Omega) \subset \mathcal{M}(\Omega)$ be the cone of nonnegative bounded Radon measure on $\Omega \subseteq \mathbb{R}^{N}(N \geq 1)$. For any $\mu \in \mathcal{M}(\Omega)$, we set

$$
\|\mu\|_{\mathcal{M}(\Omega)}:=|\mu|(\Omega)
$$

where $|\mu|$ stands for the total variation of $\mu$.

The duality map $\langle\cdot, \cdot\rangle_{\Omega}$ between the space $\mathcal{M}(\Omega)$ and $C_{c}(\Omega)$ is defined by

$$
\langle\mu, \varphi\rangle_{\Omega}=\int_{\Omega} \varphi d \mu .
$$

For any $\mu \in \mathcal{M}(\Omega)$ and any Borel set $B \subseteq \Omega$, the restriction $\mu\llcorner B$ of $\mu$ to $B$ is defined by setting

$$
(\mu\llcorner B)(A):=\mu(B \cap A) \quad \text { for every Borel set } A \subseteq \Omega \text {. }
$$

It is worth observing that $(\mu\llcorner B)(\emptyset)=0$.

$\mathcal{M}_{s}^{+}(\Omega)$ denotes the set of nonnegative measures singular with respect to the Lebesgue measure, namely

$$
\mathcal{M}_{s}^{+}(\Omega):=\left\{\mu \in \mathcal{M}^{+}(\Omega) / \exists \text { a Borel set } B \subseteq \Omega \text { such that }|B|=0, \mu=\mu\llcorner B\},\right.
$$

$|\cdot|$ denotes the Lebesgue measure on $\mathbb{R}^{N}(N \geq 1)$. Similarly, $\mathcal{M}_{a c}^{+}(\Omega)$ denotes the set of nonnegative measures absolutely continuous with respect to the Lebesgue measure, namely

$$
\mathcal{M}_{a c}^{+}(\Omega):=\left\{\mu \in \mathcal{M}^{+}(\Omega) / \mu(B)=0 \text { for every Borel set } B \subseteq \Omega \text { such that }|B|=0\right\} .
$$

Recall that $\mathcal{M}_{s}^{+}(\Omega) \cap \mathcal{M}_{a c}^{+}(\Omega)=\{0\}$. Moreover, by the Lebesgue decomposition and Radon-Nikodym theorem (see [9]), for any $\mu \in \mathcal{M}^{+}(\Omega)$ : 
(i) There exists a unique couple $\mu_{a c} \in \mathcal{M}_{a c}^{+}(\Omega), \mu_{s} \in \mathcal{M}_{s}^{+}(\Omega)$ such that

$$
\mu=\mu_{a c}+\mu_{s}
$$

(ii) There exists a unique nonnegative function $u_{r} \in L^{1}(\Omega)$ called the density of measure $\mu_{a c}$ such that

$$
\mu_{a c}(B)=\int_{B} u_{r} d x \quad \text { for every Borel set } B \subseteq \Omega
$$

Let $Q=\Omega \times(0, T), T$ is a positive constant.

$L^{\infty}\left((0, T), \mathcal{M}^{+}(\Omega)\right)$ denotes the set of nonnegative Radon measures $u \in \mathcal{M}^{+}(Q)$ which satisfy the following property: For almost every $t \in(0, T)$, there exists a measure $u(\cdot, t) \in$ $\mathcal{M}^{+}(\Omega)$ such that

(a) For every $\xi \in C(\bar{Q})$, the map $t \mapsto\langle u(\cdot, t), \xi(\cdot, t)\rangle_{\Omega}$ is Lebesgue measurable and there holds

$$
\langle u, \xi\rangle_{Q}=\int_{0}^{T}\langle u(\cdot, t), \xi(\cdot, t)\rangle_{\Omega} d t
$$

(b) For every Borel set $B \subseteq Q$, the map $t \mapsto u(\cdot, t)\left(B^{t}\right)$ is Lebesgue measurable and there holds

$$
u(B)=\int_{0}^{T} u(\cdot, t)\left(B^{t}\right) d t
$$

where $B^{t}=\{x \in \Omega /(x, t) \in B\}$.

(c) There exists a constant $C>0$ such that

$$
\text { ess } \sup _{t \in(0, T)}\|u(\cdot, t)\|_{\mathcal{M}^{+}(\Omega)} \leq C \text {. }
$$

Set

$$
\|u\|_{L^{\infty}\left((0, T), \mathcal{M}^{+}(\Omega)\right)}=\operatorname{ess} \sup _{t \in(0, T)}\|u(\cdot, t)\|_{\mathcal{M}^{+}(\Omega)} \cdot
$$

If $u \in L^{\infty}\left((0, T), \mathcal{M}^{+}(\Omega)\right)$, it is easily seen that $u_{a c}, u_{s} \in L^{\infty}\left((0, T), \mathcal{M}^{+}(\Omega)\right)$ as well and that $u_{r} \in L^{\infty}\left((0, T), L^{1}(\Omega)\right)$.

Moreover, inequality (2.4) implies that, for every $\xi \in C(\bar{Q})$,

$$
\left\langle u_{a c}, \xi\right\rangle_{Q}=\int_{Q} u_{r} \xi d x d t \text { and }\left\langle u_{s}, \xi\right\rangle_{Q}=\int_{0}^{T}\left\langle u_{s}(\cdot, t), \xi(\cdot, t)\right\rangle_{\Omega} d t
$$

(see also $[7,8])$.

\subsection{Young measures}

We denote by $C_{c}(\mathbb{R})$ the space of continuous real functionals with compact support in $\mathbb{R}$ and by $\mathcal{M}(\mathbb{R})$ the Banach space Radon measure on $\mathbb{R}$ endowed with the norm

$$
\|\mu\|_{\mathcal{M}(\mathbb{R})}:=|\mu|(\mathbb{R}) \quad \text { for any } \mu \in \mathcal{M}(\mathbb{R})
$$


By $\mathcal{M}(\mathbb{R})$ we denote the cone of positive finite Radon measure, and by $\mathcal{P}(\mathbb{R})$ the convex set of probability measure on $\mathbb{R}$ :

$$
\|\tau\|_{\mathcal{M}(\mathbb{R})}:=\tau(\mathbb{R}) \quad \text { for any } \tau \in \mathcal{P}(\mathbb{R})
$$

Clearly, $\mathcal{P}(\mathbb{R}) \subset \mathcal{M}^{+}(\mathbb{R}) \subset \mathcal{M}(\mathbb{R})$.

By a bounded Caratheodory integrand on $A \times \mathbb{R}$ we mean that any function $\varphi: A \times$ $\mathbb{R}^{N} \rightarrow \mathbb{R}$ is bounded and measurable, with $\varphi(x, \cdot)$ continuous for almost everywhere $x \in A$. The duality map $\langle\cdot, \cdot\rangle$ between the spaces $\mathcal{M}(\mathbb{R})$ and $C_{c}(\mathbb{R})$ is expressed as

$$
\langle\mu, \rho\rangle_{\mathbb{R}}:=\int_{\mathbb{R}} \rho d \mu,
$$

which can be extended to functions $\rho \in C_{c}(\mathbb{R})$. Let $A \subset \mathbb{R}^{N}(N \geq 1)$ be a bounded open set. We use the above equality to define the quantity $\langle\mu, \rho\rangle_{\mathbb{R}}$ for any $\mu \in \mathcal{M}(\mathbb{R})$ and every $\mu$-integrable function $\rho$. Similar notation will be used for the space $\mathcal{M}(A \times \mathbb{R})$ of finite Radon-measures on $A \times \mathbb{R}$. By $\mathcal{Y}(A, \mathbb{R})$ we denote the set of Young measures on $A \times \mathbb{R}$ which are defined as follows (e.g. [15]).

Definition 2.1 A Young measure on $A \times \mathbb{R}$ is a positive Radon-measure $\tau$ on $A \times \mathbb{R}$ such that

$$
\tau(E \times \mathbb{R})=|E| \text { for any Borel set } E \subseteq A \text {. }
$$

If $f \in L^{1}(A)$, the Young measure associated with $f$ is the measure $\tau \in \mathcal{Y}(A, \mathbb{R})$ such that

$$
\tau(E \times F)=\left|E \cap f^{-1}(F)\right| \quad \text { for any } E \subseteq A, F \subseteq \mathbb{R} .
$$

For any bounded Caratheodory integrand, there holds

$$
\int_{A \times \mathbb{R}} \varphi d \tau=\int_{A} \varphi(x, f(x)) d x
$$

Let us recall the following result (e.g. $[9,10,15])$.

Proposition 2.1 Let $\tau \in \mathcal{Y}(A, \mathbb{R})$. Then, for almost everywhere $x \in A$, there exists a probability measure $\tau_{x} \in \mathcal{P}(\mathbb{R})$ for any bounded Caratheodory integrand $\varphi$ on $A \times \mathbb{R}$ :

(i) The map

$$
x \mapsto\left\langle\tau_{x},\left.\varphi(x, \cdot)\right|_{\mathbb{R}}=\int_{\mathbb{R}} \varphi(x, \xi) d \tau_{x}(\xi)\right.
$$

is Lebesgue measurable;

(ii) There holds

$$
\int_{A \times \mathbb{R}} \varphi d \tau=\int_{A}\left(\int_{\mathbb{R}} \varphi(x, \xi) d \tau_{x}(\xi)\right) d x
$$


More generally, Proposition 2.1 holds true for very $\varphi: A \times \mathbb{R} \rightarrow \mathbb{R}$ measurable and nonnegative or $\tau$-integrable, we shall identify and $\tau \in \mathcal{Y}(A, \mathbb{R})$ with the associated family $\left\{\tau_{x} \mid x \in A\right\}$ which is called disintegration of $\tau$.

Remark 2.1 If $\tau$ is the Young measure associated with a function $f \in L^{1}(A)$, equalities (2.8)-(2.9) imply that

$$
\varphi(x, f(x))=\left\langle\tau_{x}, \varphi(x, \cdot)\right\rangle_{\mathbb{R}}=\int_{\mathbb{R}} \varphi(x, \xi) d \tau_{x}(\xi)
$$

for almost every $x \in A$ and for any bounded Caratheodory integrand $\varphi$ on $A \times \mathbb{R}$. Therefore,

$$
\tau_{x}=\delta_{f(x)} \quad \text { for almost every } x \in A,
$$

where $\delta_{p}$ denotes the Dirac mass concentrated in $p \in \mathbb{R}$.

The notion of narrow convergence of Young measures is as follows.

Definition 2.2 Let $\tau^{n}, \tau \in \mathcal{Y}(A, \mathbb{R})$. We say that $\tau^{n} \rightarrow \tau$ narrowly in $A \times \mathbb{R}$ if for any bounded Caratheodory integrand $\varphi: A \times \mathbb{R} \rightarrow \mathbb{R}$ there holds

$$
\int_{A \times \mathbb{R}} \varphi d \tau^{n} \rightarrow \int_{A \times \mathbb{R}} \varphi d \tau
$$

Remark 2.2 If the Young measures $\left\{\tau^{n}\right\}$ are associated with a sequence of functions $\left\{f_{n}\right\} \subseteq$ $L^{1}(A)$, then $\left\{\tau^{n}\right\}$ converge narrowly to $\tau$ if and only if

$$
\int_{A} \varphi\left(x, f_{n}(x)\right) d x=\int_{A \times \mathbb{R}} \varphi d \tau^{n} \rightarrow \int_{A \times \mathbb{R}} \varphi d \tau
$$

for any bounded Caratheodory integrand.

This convergence still holds when $\varphi$ is a Caratheodory integrand with linear growth with respect to $\xi$ (i.e. $|\varphi(x, \xi)| \leq \alpha(x)+|\xi|$, where $\alpha \in L^{1}(A)$ as soon as the sequence $\left\{f_{n}\right\}$ is uniformly integrable.

If $\tau^{n}$ and $\tau$ are the Young measures associated with the measurable functions $f_{n}$ and $f$ respectively, then $\tau^{n} \rightarrow \tau$ narrowly if and only if $f_{n} \rightarrow f$ in measure. In other words, $f_{n} \rightarrow f$ in measure if and only if the Young measure associated with $f_{n}$ is $\delta_{f(x)}($ see $[15,16])$.

Definition 2.3 A subset $\mathcal{U} \subseteq L^{1}(A, \mathbb{R})$ is said to be uniformly integrable if

(i) There exists $M>0$ such that

$$
\|f\|_{L^{1}(A, \mathbb{R})}:=\int_{A}|f(x)| d x \leq M \quad \text { for any } f \in \mathcal{U} .
$$

(ii) For $\epsilon>0$, there exists $\beta>0$ such that, for any $f \in \mathcal{U}$,

$$
|E|<\beta \quad \Rightarrow \quad \int_{E}|f(x)| d x<\epsilon
$$


Notice that for any function $f \in L^{1}(A)$ is equi-integrable if the assumption of Definition 2.3-(ii) holds true.

The following proposition is a consequence of the more general Prokhorov's theorem (e.g. see [15]).

Proposition 2.2 Let $\left\{f_{n}\right\}$ be bounded in $L^{1}(A)$ and $\left\{\tau^{n}\right\}$ be the sequence of associated Young measures. Then

(i) There exist a sequence $\left\{f_{n_{k}}\right\} \subseteq\left\{f_{n}\right\}$ and a Young measure $\tau$ on $A \times \mathbb{R}$ such that $\tau^{n_{k}} \rightarrow \tau$ narrowly in $A \times \mathbb{R}$;

(ii) For any $h \in C(\mathbb{R})$ such that the sequence $\left\{h\left(f_{n_{k}}\right)\right\}$ is bounded in $L^{1}(A)$ and is equiintegrable, there hold

$$
\int_{A}\left(\int_{\mathbb{R}}|h(\xi)| d \tau_{x}(\xi)\right) d x<+\infty \text { and } h\left(f_{n_{k}}\right) \rightarrow h^{*} \quad \text { in } L^{1}(A)
$$

where

$$
h^{*}(x)=\int_{\mathbb{R}} h(\xi) d \tau_{x}(\xi) \text { for a.e. } x \in A,
$$

$\tau_{x}$ is disintegration of the Young measure $\tau$.

(iii) For any function $\varphi: A \times \mathbb{R} \rightarrow \mathbb{R}$ measurable with $\varphi(x, \cdot)$ continuous for a.e., $x \in A$ such that the sequence $\left\{\varphi\left(x, f_{n_{k}}\right)\right\}$ is bounded in $L^{1}(A)$ and is equi-integrable, there holds

$$
\int_{A} \varphi\left(x, f_{n_{k}}(x)\right) d x \rightarrow \int_{A \times \mathbb{R}} \varphi d \tau
$$

When equi-integrability of the sequence $\left\{f_{n_{k}}\right\}$ fails, Proposition 2.2-(ii) cannot be directly used with $h(f)=f$. However, we can associate with $\left\{f_{n_{k}}\right\}$ an equi-integrable subsequence by removing sets of small measure, this is the content of the next coming proposition (e.g. see $[10,15])$.

Proposition 2.3 (Biting Young measure) Let $\left\{f_{n}\right\}$ be bounded in $L^{1}(A)$. Let $\tau \in \mathcal{Y}(A, \mathbb{R})$ and $\left\{f_{n_{k}}\right\}$ be respectively the limiting Young measure and a subsequence given in Proposition 2.3 in correspondence with $\left\{f_{n}\right\}$. Then there exist a subsequence $\left\{f_{n_{j}}\right\} \equiv\left\{f_{n_{j}}\right\} \subseteq\left\{f_{n_{k}}\right\}$ and a sequence of measurable sets $\left\{A_{j}\right\}, A_{j} \subset A, A_{j+1} \subset A_{j}$ for any $j \in \mathbb{N},\left|A_{j}\right| \rightarrow 0$ as $j \rightarrow \infty$ such that the sequence $\left\{f_{n_{j}} \chi_{A \backslash A_{j}}\right\}$ is equi-integrable. Moreover, the barycenter of the Young measure disintegration $\tau_{x}$,

$$
f_{b}(x)=\int_{\mathbb{R}^{N}} \xi d \tau_{x}(\xi) \in L^{1}(A) \quad \text { and there holds } \quad\left\{f_{n_{j}} \chi_{A \backslash A_{j}}\right\} \rightarrow f_{b} \quad \text { in } L^{1}(A) .
$$

\section{Statement of main results}

Throughout this paper, we consider the backward parabolic equation

$$
(v \cdot \alpha) \begin{cases}\xi_{t}+\alpha \xi_{x x}+f^{\prime}(+\infty) \xi=\xi_{v} & \text { in } Q, \\ \xi=0 & \text { on } \partial \Omega \times(0, T), \\ \xi(\cdot, T)=0 & \text { in } \Omega \times\{T\},\end{cases}
$$


which has a unique solution $\xi$ in $C^{1,2}(Q) \cap C^{1}(\bar{Q})$ for any $\xi_{v} \in C(Q)$ (see $[12,13,30]$ ), where

$$
C^{1,2}(Q)=\left\{\xi \in C(\bar{Q}) / \exists \xi_{t} \in C(\bar{Q}), \frac{\partial \xi}{\partial x_{i}} \in C(\bar{Q}), \frac{\partial^{2} \xi}{\partial x_{i} \partial x_{j}} \in C(\bar{Q})\right\} .
$$

Definition 3.1 For any $u_{0} \in \mathcal{M}^{+}(\Omega)$, a function $u$ is called a weak Radon measure solution of problem $(\mathrm{P})$ if the couple $(u, \tau)$ is such that

(i) $u \in L^{\infty}\left((0, T), \mathcal{M}^{+}(\Omega)\right), \tau \in \mathcal{Y}(Q, \mathbb{R})$

$$
u_{r}(x, t)=\int_{[0,+\infty)} \lambda d \tau_{(x, t)}(\lambda) \quad \text { a.e. }(x, t) \in Q
$$

(ii) $\psi\left(u_{r}\right) \in L^{1}\left((0, T), W_{0}^{1,1}(\Omega)\right)$;

(iii) For almost everywhere $(x, t) \in Q$, there hold

$$
\tau_{(x, t)}=\delta_{u_{r}(x, t)} \quad \text { and } \quad \operatorname{supp} \tau_{(x, t)} \subseteq[0,+\infty) \quad \text { a.e. }(x, t) \in Q \text {, }
$$

where we denote by $\delta_{u_{r}(x, t)}$ the Dirac mass concentrated at $u_{r}(x, t)$ and $\tau_{(x, t)} \in \mathcal{P}(\mathbb{R})$ is the disintegration of $\tau$.

(iv) For every $\xi \in C^{1}\left([0, T], C_{0}^{1}(\Omega)\right), \xi(\cdot, T)=0$ in $\Omega, u$ satisfies the identity

$$
\begin{aligned}
& \int_{Q}\left\{u_{r} \xi_{t}+\psi^{*} \xi_{x x}\right\} d x d t+\int_{0}^{T}\left\langle u_{s}(\cdot, t), \bar{\xi}_{v}(\cdot, t)\right\rangle_{\Omega} d t \\
& \quad+f^{\prime}(+\infty) \int_{0}^{T}\left\langle u_{s}(\cdot, t), \xi(\cdot, t)\right\rangle_{\Omega} d t+\int_{Q} f^{*} \xi d x d t=\left\langle u_{0}, \xi(\cdot, 0)\right\rangle_{\Omega}
\end{aligned}
$$

whenever $\bar{\xi}_{v}=\xi_{t}+\alpha \xi_{x x}$ and

$$
f^{*}(x, t)=\int_{[0,+\infty)} f(\lambda) d \tau_{(x, t)}(\lambda), \psi^{*}(x, t)=\int_{[0,+\infty)} \psi(\lambda) d \tau_{(x, t)}(\lambda)
$$

for a.e. $(x, t) \in Q$.

Remark 3.1 Since the class of test functions $\xi \in C^{1}\left([0, T], C_{0}^{1}(\Omega)\right)$ is a solution to the backward parabolic equations $(v \cdot \alpha)$ such that $\xi_{v} \in C(Q)$, then Eq. (3.3) is reduced as follows:

$$
\int_{Q}\left\{u_{r} \xi_{t}+\psi^{*} \xi_{x x}\right\} d x d t+\int_{0}^{T}\left\langle u_{s}(\cdot, t), \xi_{v}(\cdot, t)\right\rangle_{\Omega} d t+\int_{Q} f^{*} \xi d x d t=\left\langle u_{0}, \xi(\cdot, 0)\right\rangle_{\Omega},
$$

where $\xi_{v}=\xi_{t}+\alpha \xi_{x x}+f^{\prime}(+\infty) \xi$ in $Q$.

The existence solution to problem $(\mathrm{P})$ is given by the following result.

Theorem 3.1 Suppose that (1.1), (1.4), $(H),(R),(G)$, and $u_{0} \in \mathcal{M}^{+}(\Omega)$ are satisfied. Then problem $(\mathrm{P})$ has a solution $(u, \tau)$ which is obtained as a limit point of the sequence $\left\{u_{n}\right\}$ of solutions to problem $\left(P_{n}\right)$. Moreover, $(u, \tau)$ is a solution of problem $(\mathrm{P})$ in the sense of Young measures. 
Let us consider the following problem:

$$
\begin{cases}v_{t}=[\psi(v)]_{x x}+f(u) & \text { in } Q:=\Omega \times(0, T) \\ v=0 & \text { on } \partial \Omega \times(0, T), \\ v(x, 0)=0 & \text { in } \Omega .\end{cases}
$$

In view of $[12,13]$, problem $(Z)$ has a unique solution function $v$ in $L^{\infty}(Q) \cap L^{2}((0, T)$, $\left.H_{0}^{1}(\Omega)\right) \cap C(Q)$. Moreover, from Theorem 3.1, problem (Z) possesses a weak solution $v(\cdot, t) \in \mathcal{M}^{+}(\Omega)$ for a.e. $t \in(0, T)$. The estimate decay is given by the following result.

Theorem 3.2 Suppose that $(R),(G)$, and $u_{0} \in \mathcal{M}^{+}(\Omega)$ are verified. The functions $u$ and $v$ are weak solutions to problems $(\mathrm{P})$ and $(\mathrm{Z})$ respectively in the sense of Theorem 3.1. Then there holds

$$
\|u(\cdot, t)-v(\cdot, t)\|_{\mathcal{M}^{+}(\Omega)} \leq C \frac{\left\|u_{0}\right\|_{\mathcal{M}^{+}(\Omega)}}{t^{\alpha}}
$$

for any $\alpha>0$ and $C$ is a positive constant. Moreover, if we extend $t \in(0, T)$ into $(0,+\infty)$, then we obtain that the following statement

$$
\lim _{t \rightarrow+\infty}\|u(\cdot, t)-v(\cdot, t)\|_{\mathcal{M}^{+}(\Omega)}=0
$$

holds true.

Regarding the study of the asymptotic behavior of the Radon measure-valued solutions to the nonlinear parabolic equation $(\mathrm{P})$, we construct the pseudo-stationary solutions to problem $(\mathrm{P})$. To this purpose, we consider the pseudo-stationary problem as follows:

$$
\begin{cases}-[\psi(w)]_{x x}+w=u_{0}(x) & \text { in } \Omega, \\ w=0 & \text { on } \partial \Omega\end{cases}
$$

where $u_{0} \in \mathcal{M}^{+}(\Omega)$ and the function $\psi$ verifies hypothesis (G). Notice that the nonlinear elliptic equation (S) admits a nonnegative Radon measure-valued solution i.e. $w \in \mathcal{M}^{+}(\Omega)$.

The main goal of the asymptotic behavior of the Radon measure-valued solutions to problem $(\mathrm{P})$ is given in the following theorem.

Theorem 3.3 Assume that hypotheses (1.1), $(R),(G)$, and $u_{0} \in \mathcal{M}^{+}(\Omega)$ are fulfilled. $w$ is a pseudo-stationary Radon measure-valued solution obtained from problem $(S)$ and $u$ is a Radon measure-valued solution in the sense of Theorem 3.1 such that

$$
\limsup _{t \rightarrow+\infty}\|u(\cdot, t)\|_{\mathcal{M}^{+}(\Omega)}<\infty
$$

Then

$$
u(\cdot, t) \rightarrow w \quad \text { in } \mathcal{M}^{+}(\Omega) \quad \text { as } t \rightarrow+\infty .
$$


Remark 3.2 Before we prove assertion (3.8), we shall ensure that statement (3.7) holds true. Indeed, the function $u$ is a Radon measure-valued solution to problem $(\mathrm{P})$, then $u(\cdot, t) \in \mathcal{M}^{+}(\Omega)$ for a.e. $t \in(0, T)$. By the extension of the solution to global solutions on $\Omega \times(0,+\infty)$, we infer that $\|u(\cdot, t)\|_{\mathcal{M}^{+}(\Omega)} \leq C$ for a.e. $t \in(0,+\infty)$ so that (3.8) is obtained.

\section{Approximating problems}

To prove the existence, decay estimate, and the asymptotic behavior of the solutions, we consider the approximating problem $P_{n}$ as follows:

$$
\begin{cases}u_{n t}=\left[\psi_{n}\left(u_{n}\right)\right]_{x x}+f\left(u_{n}\right) & \text { in } Q, \\ u_{n}=0 & \text { on } \partial \Omega \times(0, T), \\ u_{n}(x, 0)=u_{0 n} & \text { in } \Omega .\end{cases}
$$

The approximating function $\psi_{n}$ is such that

$$
\psi_{n}(s)=\psi(s)+\frac{1}{n}
$$

Since $u_{0} \in \mathcal{M}^{+}(\Omega)$, then the approximation of the Radon measure $u_{0}$ is given by [7, Lemma 4.1], such that $\left\{u_{0 n}\right\} \subseteq C_{0}^{\infty}(\Omega)$ satisfies

$$
\left\{\begin{array}{l}
u_{0 n} \stackrel{*}{\rightarrow} u_{0} \quad \text { in } \mathcal{M}^{+}(\Omega) \\
u_{0 n} \rightarrow u_{0 r} \quad \text { a.e. in } \Omega \\
\left\|u_{0 n}\right\|_{L^{1}(\Omega)} \leq\left\|u_{0}\right\|_{\mathcal{M}^{+}(\Omega)}
\end{array}\right.
$$

Notice that the use of $L^{1}$ - norm of the initial data is a consequence of the above [7, Lemma 4.1].

The existence of a weak solution $u_{n}$ in $C\left((0, T), L^{1}(\Omega)\right) \cap L^{2}\left((0, T), H_{0}^{1}(\Omega)\right) \cap L^{\infty}(Q)$ of problem $\left(P_{n}\right)$ is ensured by [4, Theorem 5] and [21, Chapter V, Theorem 2.1 and 6.7]. Moreover, $u_{n t} \in L^{2}\left((0, T), H^{-1}(\Omega)\right)$ given by [22, Proposition 6.1] and $u_{n} \in L^{\infty}(Q)$ is proved in [23, Theorem 3.1]. Then a definition of the weak solution $\left\{u_{n}\right\} \subseteq C^{\infty}(\bar{Q})$ of $\left(P_{n}\right)$ satisfies the following statement:

$$
\int_{0}^{T}\left\langle u_{n}(\cdot, t), \xi_{t}(\cdot, t)\right\rangle_{\Omega} d t=\int_{0}^{T}\left\langle\left[\psi\left(u_{n}\right)\right]_{x}, \xi_{x}\right\rangle_{\Omega} d t-\int_{Q} f\left(u_{n}\right) \xi d x d t-\left\langle u_{0 n}, \xi(\cdot, 0)\right\rangle_{\Omega}
$$

for every $\xi$ in $C^{1}(\bar{Q})$ such that $\xi(\cdot, T)=0$ and $\xi=0$ on $\partial \Omega \times(0, T)$.

Now we establish some technical estimates which will be used in the proof of the existence solution.

Proposition 4.1 Suppose that $f \geq 0$ in $\mathbb{R}_{+}$and $(G)$ holds. Moreover, $u_{0 n} \geq 0$ in $\Omega$. Then there holds

$$
u_{n} \geq 0 \quad \text { in } Q \text {. }
$$


Proof Since $\psi\left(u_{n}\right)=\alpha u_{n}+\beta \varphi\left(u_{n}\right)$. Let us consider the nonlinear parabolic boundary value problem

$$
\begin{cases}\mathcal{L} u_{n} \equiv u_{n t}-\operatorname{div}\left(\left(\alpha+\beta \varphi^{\prime}\left(u_{n}\right)\right) u_{n x}\right)=f\left(u_{n}\right) & \text { in } Q \\ u_{n}=0 & \text { on } \partial \Omega \times(0, T), \\ u_{n}(x, 0)=u_{0 n} & \text { in } \Omega .\end{cases}
$$

According to [21, Chapter V, Theorem 2.1 and Theorem 6.7], problem $P_{n}$ possesses a unique solution $u_{n}$. Since $u_{0 n} \geq 0$ in $\Omega$, and $f\left(u_{n}\right) \geq 0$ in $Q$, it follows that

$$
\begin{cases}\mathcal{L} u_{n} \geq 0 & \text { in } Q \\ u_{n}=0 & \text { on } \partial \Omega \times(0, T) \\ u_{n}(x, 0) \geq 0 & \text { in } \Omega\end{cases}
$$

By [25, Chap. 10, Theorem 10.1], the comparison maximum principle theorem, we obtain $u_{n}(x, t) \geq 0$ in $\bar{Q}$, whence estimate (4.4) holds.

Lemma 4.1 Assume that $(R)$ and $(G)$ are satisfied and $u_{0} \in \mathcal{M}^{+}(\Omega)$. For every $t \in[0, T]$, there holds

$$
\left\|u_{n}(\cdot, t)\right\|_{L^{1}(\Omega)} \leq e^{L t}\left\|u_{0}\right\|_{\mathcal{M}^{+}(\Omega)}
$$

where $L:=\left\|f^{\prime}\left(u_{n}\right)\right\|_{L^{\infty}\left(\mathbb{R}_{+}\right)}$is a positive constant.

Proof Let us consider the boundary value problem

$$
\mathcal{P} u_{n} \equiv\left[\psi\left(u_{n}\right)\right]_{x x}-u_{n t}+f\left(u_{n}\right)=0 \quad \text { in } Q .
$$

By Proposition 4.1, $\psi\left(u_{n}\right) \geq 0$ in $Q$ and $\psi\left(u_{n}\right)=0$ on $\partial \Omega \times(0, T)$. In view of [21, Chapter $\mathrm{V}]$, the maximum principle theorem, then for arbitrary point $\left(x_{0}, t_{0}\right) \in \partial \Omega \times(0, T)$, we obtain

$$
\frac{\partial \psi\left(u_{n}\right)}{\partial v}\left(x_{0}, t_{0}\right) \leq 0
$$

the normal outer derivative of $\psi\left(u_{n}\right)\left(x_{0}, t_{0}\right)$ at $\partial \Omega \times(0, T)$. Applying Green's formula for every $(x, t) \in Q$, there holds

$$
\int_{Q}\left[\psi\left(u_{n}\right)\right]_{x x} d x=\int_{\partial \Omega \times(0, T)} \frac{\partial \psi\left(u_{n}\right)}{\partial v} d \mathcal{H} \leq 0,
$$

where $\mathcal{H}$ denotes the Hausdorff $(N-1)$-dimensional measure. Using the Eq. (4.6), assumption (4.2), and the mean-value theorem, we deduce that

$$
\int_{\Omega} u_{n}(\cdot, t) d x \leq\left\|u_{0}\right\|_{\mathcal{M}^{+}(\Omega)}+\left\|f^{\prime}\left(u_{n}\right)\right\|_{L^{\infty}\left(\mathbb{R}_{+}\right)} \int_{0}^{t} \int_{\Omega} u_{n}(x, s) d x d s
$$

By Gronwall's inequality, estimate (4.5) is achieved. 
Proposition 4.2 Assume that hypotheses $(R)$ and $(G)$ are satisfied. Let $u_{n}$ be the solution of $\left(P_{n}\right)$. Then there hold

$$
\begin{aligned}
& \left\|\psi\left(u_{n}\right)\right\|_{L^{1}(Q)} \leq C . \\
& \left\|\left[\psi\left(u_{n}\right)\right]_{x}\right\|_{L^{2}(Q)} \leq C . \\
& \text { The sequence }\left\{\left[\psi\left(u_{n}\right)\right]_{t}\right\} \text { is bounded in } L^{1}\left((0, T), H^{-1}(\Omega)\right)+L^{1}(Q) . \\
& \left\|\psi\left(u_{n}\right)\right\|_{L^{2}\left((0, T), H_{0}^{1}(\Omega)\right)} \leq C .
\end{aligned}
$$

Proof By the definition of $\psi$ in (1.1), $\psi\left(u_{n}\right)=\alpha u_{n}+\beta \varphi\left(u_{n}\right)$. By (4.5), $u_{n}$ is bounded in $L^{1}(Q)$, and assumption $(\mathrm{G})$, it is obvious that there exists a constant $C>0$ such that (4.8) is achieved.

Since $\psi\left(u_{n}\right) \geq 0$ in $Q$ and $\psi\left(u_{n}\right)=0$ on $\partial \Omega \times(0, T)$. The fact that $u_{n}=\psi\left(\psi^{-1}\left(u_{n}\right)\right) \in$ $C^{1}\left([0, T], H_{0}^{1}(\Omega)\right)$. Let us consider the function sign defined in the following manner:

$$
\operatorname{sign}(s)= \begin{cases}1 & \text { if } s>0 \\ 0 & \text { if } s=0 \\ -1 & \text { if } s<0\end{cases}
$$

Assume that, for every $K>0, T_{K} \in C^{1}\left(\mathbb{R}_{+}\right) \cap L^{\infty}\left(\mathbb{R}_{+}\right)$such that $1 \leq T_{K}^{\prime}(s) \leq 2$ in $\mathbb{R}_{+}, T_{K}(0)=$ 0 and $T_{K}(s) \rightarrow \frac{3}{2} s \chi_{(1,1+\epsilon)}(s)$ as $K \rightarrow+\infty$ for every $0<\epsilon<1$, where $\chi_{(1,1+\epsilon)}$ is a characteristic function on $(1,1+\epsilon)$.

For instance, let us consider $\left\{g_{K}\right\} \subseteq C^{1}(\mathbb{R})$ such that $g_{K}(s) \rightarrow \operatorname{sign}(s)$ as $K \rightarrow+\infty$ for every $s \neq 0$ to be any sequence satisfying the following conditions: $g_{K}(0)=0,\left|g_{K}(s)\right| \leq 1$, $g_{K}^{\prime}(s) \geq 0,\left|s g_{K}(s)\right| \leq 1$ for every $s \in \mathbb{R}$, and $g_{K}^{\prime}(s)=0$ if $|s| \geq \frac{1}{K}$. By recalling the sequence $\left\{g_{K}\right\}$ constructed in [1], we can construct the sequence of the function $\left\{T_{K}\right\} \subseteq C^{1}\left(\mathbb{R}_{+}\right) \cap L^{\infty}\left(\mathbb{R}_{+}\right)$ such that $T_{K}(s)=\left(1+\frac{1}{2} g_{K}(s)\right) s \chi_{(1,1+\epsilon)}(s)$.

Assume that $T_{K}\left(\psi\left(u_{n}\right)\right)$ is a test function to the approximation problem $\left(P_{n}\right)$. Then we get

$$
\begin{aligned}
\int_{Q}\left|\left[\psi\left(u_{n}\right)\right]_{x}\right|^{2} T_{K}^{\prime}\left(\psi\left(u_{n}\right)\right) d x d t \\
=\int_{\Omega}\left(\int_{0}^{u_{0 n}(x)} T_{K}(\psi(s)) d s\right) d x \\
\quad-\int_{\Omega}\left(\int_{0}^{u_{n}(x, T)} T_{K}(\psi(s)) d s\right) d x+\int_{Q} f\left(u_{n}\right) T_{K}\left(\psi\left(u_{n}\right)\right) d x d t .
\end{aligned}
$$

Since $1 \leq T_{K}^{\prime}\left(\psi\left(u_{n}\right)\right) \leq 2$ and $T_{K}\left(\psi\left(u_{n}\right)\right), T_{K}(\psi(s)) \in L^{\infty}\left(\mathbb{R}_{+}\right)$for every $K$, then (4.12) yields

$$
\begin{aligned}
\int_{Q}\left|\left[\psi\left(u_{n}\right)\right]_{x}\right|^{2} d x d t \leq & \left\|T_{K}\left(\psi\left(u_{n}\right)\right)\right\|_{L^{\infty}\left(\mathbb{R}_{+}\right)}\left\|u_{0}\right\|_{\mathcal{M}^{+}(\Omega)} \\
& +\left\|T_{K}\left(\psi\left(u_{n}\right)\right)\right\|_{L^{\infty}\left(\mathbb{R}_{+}\right)}\left\|f\left(u_{n}\right)\right\|_{L^{\infty}\left(\mathbb{R}_{+}\right)}|Q| .
\end{aligned}
$$

Then there exists a positive constant $C=C\left(\left\|u_{0}\right\|_{\mathcal{M}^{+}(\Omega)},\left\|f\left(u_{n}\right)\right\|_{L^{\infty}\left(\mathbb{R}_{+}\right)},|Q|\right)>0$ such that

$$
\int_{Q}\left|\left[\psi\left(u_{n}\right)\right]_{x}\right|^{2} d x d t \leq C
$$


Assume that $\xi \psi^{\prime}\left(u_{n}\right)$ is a test function into the first equation of $\left(P_{n}\right)$. Then we have

$$
\int_{Q} \psi\left(u_{n}\right) \xi_{t} d x d t=-\int_{Q}\left[\psi\left(u_{n}\right)\right]_{x}\left[\xi \psi^{\prime}\left(u_{n}\right)\right]_{x} d x d t+\int_{Q} f\left(u_{n}\right) \psi^{\prime}\left(u_{n}\right) \xi d x d t
$$

for any $\xi \in C_{c}^{1}(Q)$. Let us estimate each term of the right-hand side of Eq. (4.13). To this purpose, we consider its first term

$$
\begin{aligned}
\int_{Q}\left[\psi\left(u_{n}\right)\right]_{x}\left[\xi \psi^{\prime}\left(u_{n}\right)\right]_{x} d x d t= & \int_{Q}\left[\psi\left(u_{n}\right)\right]_{x} \psi^{\prime}\left(u_{n}\right) \xi_{x} d x d t \\
& \left.+\int_{Q}\left(\left[\psi\left(u_{n}\right)\right]_{x}\right)^{2} \psi^{\prime \prime}\left(u_{n}\right)\right) \xi d x d t
\end{aligned}
$$

By hypothesis $(G), \psi^{\prime}\left(u_{n}\right), \psi^{\prime \prime}\left(u_{n}\right) \in L^{\infty}\left(\mathbb{R}_{+}\right)$, then we obtain

$$
\begin{aligned}
\int_{Q}\left[\psi\left(u_{n}\right)\right]_{x}\left[\xi \psi^{\prime}\left(u_{n}\right)\right]_{x} d x d t \leq & \left\|\psi^{\prime}\left(u_{n}\right)\right\|_{L^{\infty}\left(\mathbb{R}_{+}\right)} \int_{Q}\left[\psi\left(u_{n}\right)\right]_{x} \xi_{x} d x d t \\
& +\left\|\psi^{\prime \prime}\left(u_{n}\right)\right\|_{L^{\infty}(\mathbb{R})} \int_{Q}\left|\left[\psi\left(u_{n}\right)\right]_{x}\right|^{2} \xi d x d t
\end{aligned}
$$

By (4.9) and applying Holder's inequality, there exist two positive constants $C_{0}$ and $C_{1}$ such that

$$
\left|\int_{Q}\left[\psi\left(u_{n}\right)\right]_{x}\left[\xi \psi^{\prime}\left(u_{n}\right)\right]_{x} d x d t\right| \leq C_{0}\left\|\xi_{x}\right\|_{L^{2}(Q)}+C_{1}\|\xi\|_{L^{\infty}(Q)}
$$

On the other hand, we consider the second term on the right-hand side of Eq. (4.13). From assumptions (G) and (R), we deduce that

$$
\left|\int_{Q} f\left(u_{n}\right) \psi^{\prime}\left(u_{n}\right) \xi d x d t\right| \leq C_{2}\|\xi\|_{L^{\infty}(Q)}
$$

where $C_{2}$ is a positive constant. Hence the boundedness of the sequence $\left\{\left[\psi\left(u_{n}\right)\right]_{t}\right\}$ in $L^{2}\left((0, T), H^{-1}(\Omega)\right)+L^{1}(Q)$ follows.

To end this proof, it remains to establish estimate (4.11), let us consider the function $h$ defined by

$$
h(s)= \begin{cases}0 & \text { if } 0 \leq s \leq t_{1} \\ s-t_{1} & \text { if } t_{1} \leq s \leq t_{2} \\ t_{2}-t_{1} & \text { if } s \geq t_{2}\end{cases}
$$

for any $t_{1}, t_{2} \in(0, T)$ such that $t_{1}+1<t_{2}$, and we observe that $0 \leq h(s)<C, s \in\left(0, t_{2}\right)$. By assumption (G), it is easy to observe that for every $n \in \mathbb{N}\left[\psi\left(u_{n}\right)\right]_{s}=0$ on $\partial \Omega \times(0, T)$ for the homogeneous Dirichlet condition. Multiplying the approximation problem $\left(P_{n}\right)$ by the test function $h(s)\left[\psi\left(u_{n}\right)\right]_{s}$ and integrating over $\Omega \times\left(0, t_{2}\right)$, we obtain

$$
\begin{aligned}
0 & \leq \int_{0}^{t_{2}} h^{\prime}(s) d s \int_{\Omega} \psi^{\prime}\left(u_{n}\right)\left(u_{n s}\right)^{2} d x \\
& =-\frac{1}{2} h\left(t_{2}\right) \int_{\Omega}\left|\left[\psi\left(u_{n}\right)\right]_{x}\right|^{2}\left(x, t_{2}\right) d x
\end{aligned}
$$




$$
+\frac{1}{2} \int_{0}^{t_{2}} h^{\prime}(s) d s \int_{\Omega}\left|\left[\psi\left(u_{n}\right)\right]_{x}\right|^{2}\left(x, t_{2}\right) d x+\int_{0}^{t_{2}} \int_{\Omega} h(s) f\left(u_{n}\right)\left[\psi\left(u_{n}\right)\right]_{s} d s d x
$$

It implies that

$$
\begin{aligned}
\int_{\Omega}\left|\left[\psi\left(u_{n}\right)\right]_{x}\right|^{2}\left(x, t_{2}\right) d x \leq & \int_{0}^{t_{2}} \int_{\Omega}\left|\left[\psi\left(u_{n}\right)\right]_{x}\right|^{2}\left(x, t_{2}\right) d x \\
& +2 \int_{0}^{t_{2}} \int_{\Omega} h(s) f\left(u_{n}\right)\left[\psi\left(u_{n}\right)\right]_{s} d s d x
\end{aligned}
$$

In view of assumption ( $R), f\left(u_{n}\right) \in L^{\infty}\left(\mathbb{R}_{+}\right)$, and the fact that (4.10) is satisfied, the last term on the right-hand side of the previous estimate (4.18) is bounded. Since (4.9) holds, there is a positive constant $C$ such that (4.11) is achieved.

\section{Existence result}

Now we study the limit points of the sequences $\left\{u_{n}\right\}$ and $\psi\left(u_{n}\right)$ as $n \rightarrow \infty$.

Proposition 5.1 Suppose that (1.1), (R), and $(G)$ are satisfied. Let $u_{n}$ be the solution of the approximating problem $\left(P_{n}\right)$. Then there exist a subsequence $\left\{u_{n_{k}}\right\} \subseteq\left\{u_{n}\right\}, v \in$ $L^{2}\left((0, T), H_{0}^{1}(\Omega)\right) \cap L^{\infty}\left((0, T), H_{0}^{1}(\Omega)\right) \cap L^{\infty}(Q)$ and $v_{t} \in L^{2}(Q)$ such that

$$
\begin{aligned}
& \psi\left(u_{n_{k}}\right) \rightarrow v \quad \text { in } L^{1}(Q), \\
& {\left[\psi\left(u_{n_{k}}\right)\right]_{x} \rightarrow v_{x} \quad \text { in } L^{2}(Q),} \\
& {\left[\psi\left(u_{n_{k}}\right)\right]_{t} \rightarrow v_{t} \quad \text { in } L^{2}(Q),} \\
& \psi\left(u_{n_{k}}\right) \rightarrow v \quad \text { a.e.in } Q .
\end{aligned}
$$

Proof By using Holder's inequality

$$
\begin{aligned}
\int_{Q}\left|\left[\psi\left(u_{n}\right)\right]_{x}\right| d x d t & \leq\left[\int_{Q} \frac{\left|\left[\psi\left(u_{n}\right)\right]_{x}\right|^{2}}{\left(1+\psi\left(u_{n}\right)\right)^{2}} d x d t\right]^{\frac{1}{2}}\left[\int_{Q}\left(1+\psi\left(u_{n}\right)\right)^{2} d x d t\right]^{\frac{1}{2}} \\
& \leq C\left[\int_{Q}\left|\left[\psi\left(u_{n}\right)\right]_{x}\right|^{2} d x d t\right]^{\frac{1}{2}} .
\end{aligned}
$$

From estimate (4.9), there exists a positive constant $C>0$ such that

$$
\int_{Q}\left|\left[\psi\left(u_{n}\right)\right]_{x}\right| d x d t \leq C
$$

According to Proposition 4.2, assumption (G), and (4.8), we infer that

$$
\left\|\psi\left(u_{n}\right)\right\|_{B V(Q)}=\left\|\psi\left(u_{n}\right)\right\|_{L^{1}(Q)}+\left\|\left[\psi\left(u_{n}\right)\right]_{x}\right\|_{L^{1}(Q)}+\left\|\left[\psi\left(u_{n}\right)\right]_{t}\right\|_{L^{1}(Q)} \leq C .
$$

By [43, Chap. 3, Sect. 3.1, Theorem 3.23, and Theorem 3.9], convergence (5.1) holds. However, convergence (5.2) is the consequence of estimate (4.9). By Proposition 4.2, the sequence $\left\{\left[\psi\left(u_{n}\right)\right]_{t}\right\}$ is bounded in $L^{2}\left((0, T), H^{-1}(\Omega)\right)+L^{1}(Q)$, then there exist a subsequence denoted again $\left\{u_{n_{k}}\right\} \subseteq\left\{u_{n}\right\}$ and $v^{*} \in L^{2}\left((0, T), H_{0}^{1}(\Omega)\right) \cap L^{\infty}(Q)$ such that

$$
\psi\left(u_{n_{k}}\right) \rightarrow v^{*} \quad \text { a.e. in } Q
$$


(see [26, Proposition 4.2]). Furthermore, by [7, Proposition 5.1] (5.3) holds true, and then we have

$$
\psi\left(u_{n_{k}}\right) \rightarrow v \quad \text { a.e. in } Q
$$

with $v=v^{*}$, which leads to (5.4) being satisfied.

Proposition 5.2 Assume that (1.1), $(R)$, and $(G)$ are satisfied. Let $u_{n}$ be the weak solution of problem $\left(P_{n}\right)$. Then there exist a subsequence $\left\{u_{n_{k}}\right\}$ and $u \in L^{\infty}\left((0, T), \mathcal{M}^{+}(\Omega)\right)$ such that

$$
u_{n_{k}} \stackrel{*}{\rightarrow} u \quad \text { in } \mathcal{M}^{+}(Q) \text {. }
$$

Moreover, there exists a decreasing sequence $\left\{A_{k}\right\} \subset Q$ of Lebesgue measurable sets with $\left|A_{k}\right| \rightarrow 0$ as $k \rightarrow \infty$ such that

$$
u_{n_{k}} \chi_{Q \backslash A_{k}} \rightarrow u_{b}:=\int_{[0,+\infty)} \lambda d \tau_{(x, t)}(\lambda) \quad \text { in } L^{1}(Q),
$$

where $\tau \in \mathcal{Y}(Q, \mathbb{R})$ is the Young measure associated with $\left\{u_{n_{k}}\right\}$ and

$$
u_{n_{k}} \stackrel{*}{\rightarrow} \mu:=u-u_{b} \quad \text { in } \mathcal{M}^{+}(Q) .
$$

Proof By (4.5) and Proposition 4.2, we apply the compactness theorem given by [27], then there exist $u \in \mathcal{M}^{+}(Q)$ and a subsequence $\left\{u_{n_{k}}\right\}$ such that $u_{n_{k}} \stackrel{*}{\rightarrow} u$ in $\mathcal{M}^{+}(Q)$. As argued in $[1,10,28]$, we obtain $u \in L^{\infty}\left((0, T), \mathcal{M}^{+}(\Omega)\right)$. Since (4.5) and the compactness result implies that $\left\{u_{n_{k}}\right\}$ is bounded in $L^{1}(Q)$. By Proposition 2.2, there exist a sequence of $\left\{u_{n_{k}}\right\} \subseteq$ $\left\{u_{n}\right\}$ and a Young measure $\tau \in \mathcal{Y}(Q, \mathbb{R})$, and from Proposition 2.3 [Bitting Theorem], there exists a sequence of measure sets $A_{k} \subseteq Q, A_{k} \subseteq A_{k+1}$ and $\left|A_{k}\right| \rightarrow 0$ such that

$$
u_{n_{k}} \chi_{Q \backslash A_{k}} \rightarrow u_{b}:=\int_{[0,+\infty)} \lambda d \tau_{(x, t)}(\lambda) \quad \text { in } L^{1}(Q),
$$

where $u_{b} \in L^{1}(Q), u_{b} \geq 0$ is a barycenter of the limiting Young measure $\tau$ associated with the subsequence $\left\{u_{n_{k}}\right\}$. Moreover, repeating the same proof, we show that $\operatorname{supp} \tau_{(x, t)} \subseteq$ $[0,+\infty)$ and $\tau_{(x, t)}=\tau_{(x, t)}\left\llcorner[0,+\infty)\right.$ for almost everywhere $(x, t) \in Q$, where $\tau_{(x, t)}$ is the disintegration of the Young measure $\tau$.

By (4.5) and the compactness result, the sequence $\left\{u_{n_{k}} \chi_{Q \backslash A_{k}}\right\}$ is uniformly bounded in $L^{1}(Q)$. Therefore, there exists a Radon measure $\mu \in \mathcal{M}^{+}(Q)$ such that $u_{n_{k}} \stackrel{*}{\rightarrow} \mu$ in $\mathcal{M}^{+}(Q)$. Finally, the sequence $u_{n_{k}}$ is of $u_{n_{k}}=u_{n_{k}} \chi_{A_{k}}+u_{n_{k}} \chi_{Q \backslash A_{k}} \stackrel{*}{\rightarrow} \mu+u_{b}$ in $\mathcal{M}^{+}(Q)$. Hence $\mu:=$ $u-u_{b}$ in $\mathcal{M}^{+}(Q)$ holds true.

Proposition 5.3 Let $u_{n_{k}}, u_{b} \in L^{\infty}\left((0, T), L^{1}(\Omega)\right)$ and $\mu \in L^{\infty}\left((0, T), \mathcal{M}^{+}(\Omega)\right)$ be respectively the subsequence, function, and the measure given in Proposition 5.2. Then there exist a zero Lebesgue measure set $\mathcal{N} \subset(0, T)$ and the subsequence (denoted again $\left\{u_{n_{k}}\right\}$ such that, for any $t \in(0, T) \backslash \mathcal{N}$, there holds

$$
u_{n_{k}}(\cdot, t) \stackrel{*}{\rightarrow} \mu(\cdot, t)+u_{b}(\cdot, t) \quad \text { in } \mathcal{M}^{+}(\Omega) .
$$


Moreover, we get

$$
u_{n_{k}}(\cdot, t) \stackrel{*}{\rightarrow} u(\cdot, t) \quad \text { in } \mathcal{M}^{+}(\Omega)
$$

where $u(\cdot, t)=\mu(\cdot, t)+u_{b}(\cdot, t)$ for any $t \in(0, T) \backslash \mathcal{N}$.

Proof This proof is similar to [1, Proposition 7.4], we argue this proof in two steps:

Step 1. Assume that $h \in C^{2}\left(\mathbb{R}_{+}\right)$is such that

$$
\begin{cases}h(s) \geq 0 & \text { if } s \geq 0 \\ h(s)=s-\varphi(s) & \text { if } s \geq 1\end{cases}
$$

For every $\rho \in C_{c}^{2}(\Omega)$, set

$$
U_{h, k}^{\rho}(t):=\int_{\Omega} h\left(u_{n_{k}}\right)(x, t) \rho(x) d x .
$$

Let us prove that

$$
\lim _{k \rightarrow \infty} \int_{0}^{T}\left|U_{h, k}^{\rho}(t)-\int_{\Omega} h^{*}(x, t) \rho(x) d x-\langle\mu(\cdot, t), \rho\rangle_{\Omega}\right| d t=0
$$

where $h^{*} \in L^{\infty}\left((0, T), L^{1}(\Omega)\right)$ is defined by

$$
h^{*}(x, t)=\int_{[0,+\infty)} h(\lambda) d \tau_{(x, t)}(\lambda) \quad \text { a.e. }(x, t) \in Q
$$

By (4.5), we obtain that

$$
\left\|U_{h, k}^{\rho}(t)\right\|_{L^{\infty}(0, T)} \leq C\|\rho\|_{L^{\infty}(\Omega)}\left\|u_{n_{k}}\right\|_{L^{\infty}\left((0, T), L^{1}(\Omega)\right)} .
$$

The purpose of this step is to prove $U_{h, k}^{\rho} \in W^{1,1}(0, T)$ for every $k$.

In fact, the weak derivative of $U_{h, k}^{\rho}$ is given by

$$
\frac{d}{d t} U_{h, k}^{\rho}(t)=-\int_{\Omega}\left[\psi\left(u_{n_{k}}\right)\right]_{x}\left[\rho(x) h^{\prime}\left(u_{n_{k}}\right)\right]_{x} d x+\int_{\Omega} f\left(u_{n_{k}}\right) h^{\prime}\left(u_{n_{k}}\right) \rho(x) d x .
$$

Hence, there holds

$$
\begin{aligned}
\int_{0}^{T}\left|\frac{d}{d t} U_{h, k}^{\rho}(t)\right| d t \leq & \int_{0}^{T}\left|\int_{\Omega}\left[\psi\left(u_{n_{k}}\right)\right]_{x}\left[\rho(x) h^{\prime}\left(u_{n_{k}}\right)\right]_{x} d x\right| d t \\
& +\int_{0}^{T}\left|\int_{\Omega} f\left(u_{n_{k}}\right) h^{\prime}\left(u_{n_{k}}\right) \rho(x) d x\right| d t .
\end{aligned}
$$

Since $h^{\prime}$ is bounded and $h^{\prime \prime}$ is compactly supported in $\mathbb{R}_{+}$, by (4.8), (4.9), (4.11), and assumption (G), we may estimate each term of (5.15), then one has

$$
\begin{aligned}
\int_{0}^{T}\left|\int_{\Omega}\left[\psi\left(u_{n_{k}}\right)\right]_{x}\left[\rho(x) h^{\prime}\left(u_{n_{k}}\right)\right]_{x} d x\right| d t \leq & \int_{Q}\left|\left[\psi\left(u_{n_{k}}\right)\right]_{x}\right|\left|h^{\prime}\left(u_{n_{k}}\right)\right|\left|\rho^{\prime}(x)\right| d x d t \\
& +\int_{Q}\left|\left[\psi\left(u_{n_{k}}\right)\right]_{x}\right|^{2} \frac{h^{\prime \prime}\left(u_{n_{k}}\right)}{\psi^{\prime}\left(u_{n_{k}}\right)} d x d t .
\end{aligned}
$$


It follows that

$$
\begin{aligned}
& \int_{0}^{T}\left|\int_{\Omega}\left[\psi\left(u_{n_{k}}\right)\right]_{x}\left[\rho(x) h^{\prime}\left(u_{n_{k}}\right)\right]_{x} d x\right| d t \\
& \leq\left\|h^{\prime}\left(u_{n_{k}}\right)\right\|_{L^{\infty}\left(\mathbb{R}_{+}\right)} \int_{Q}\left|\left[\psi\left(u_{n_{k}}\right)\right]_{x}\right|\left|\rho^{\prime}(x)\right| d x d t \\
& \quad+C(\beta) \int_{Q}\left|\left[\psi\left(u_{n_{k}}\right)\right]_{x}\right|^{2} \frac{\left|\psi^{\prime \prime}\left(u_{n_{k}}\right)\right|}{\psi^{\prime}\left(u_{n_{k}}\right)} d x d t .
\end{aligned}
$$

By assumption (G)-(iii) and (4.9), there exists a positive constant $C_{\rho}=C(\rho)$ such that

$$
\int_{0}^{T}\left|\int_{\Omega}\left[\psi\left(u_{n_{k}}\right)\right]_{x}\left[\rho(x) h^{\prime}\left(u_{n_{k}}\right)\right]_{x} d x\right| d t \leq C_{\rho}
$$

On the other hand, we have

$$
\int_{0}^{T}\left|\int_{\Omega} f\left(u_{n_{k}}\right) h^{\prime}\left(u_{n_{k}}\right) \rho(x) d x\right| d t \leq L\|\rho\|_{L^{\infty}(\Omega)}\left\|h^{\prime}\left(u_{n_{k}}\right)\right\|_{L^{\infty}\left(\mathbb{R}_{+}\right)} \int_{Q} u_{n_{k}} d x d t
$$

Accordingly, there exists a positive constant $\widetilde{C}_{\rho}=\widetilde{C}(\rho)$ such that

$$
\int_{0}^{T}\left|\int_{\Omega} f\left(u_{n_{k}}\right) h^{\prime}\left(u_{n_{k}}\right) \rho(x) d x\right| d t \leq \widetilde{C}_{\rho}
$$

In view of (5.15)-(5.18), the sequence $\left\{U_{h, k}^{\rho}\right\}$ is uniformly bounded in $W^{1,1}(0, T)$, whence relatively compact in $L^{1}(0, T)$. In particular, there exists a subsequence $\left\{U_{h, k_{j}}^{\rho}\right\}$ depending on $\rho$ and $h$, where a function $U_{h}^{\rho} \in L^{1}(0, T)$ is such that

$$
U_{h, k_{j}}^{\rho} \rightarrow U_{h}^{\rho} \quad \text { in } L^{1}(0, T) \quad \text { as } j \rightarrow \infty
$$

Since we have

$$
\varphi(s)=s-h(s) \quad\left(s \in \mathbb{R}_{+}\right),
$$

where $\varphi \in C\left(\mathbb{R}_{+}\right) \cap L^{\infty}\left(\mathbb{R}_{+}\right)$(see assumption $(H)$ ). By Proposition 2.2, we have

$$
\varphi\left(u_{n_{k}}\right) \stackrel{*}{\rightarrow} \varphi^{*} \quad \text { in } L^{\infty}(Q),
$$

where $\varphi^{*}$ is defined by

$$
\varphi^{*}(x, t)=\int_{[0,+\infty)} \varphi(\lambda) d \tau_{(x, t)}(\lambda) \quad(\text { a.e. }(x, t) \in Q) .
$$

In particular, combining (5.21) with (5.8), one has

$$
h\left(u_{n_{k}}\right)=u_{n_{k}}-\varphi\left(u_{n_{k}}\right) \stackrel{*}{\rightarrow}\left(u_{b}+\mu\right)-\varphi^{*} \quad \text { in } \mathcal{M}^{+}(Q),
$$


where $u_{b} \in L^{\infty}\left((0, T), L^{1}(\Omega)\right)$ and $\mu \in L^{\infty}\left((0, T), \mathcal{M}^{+}(\Omega)\right)$ are respectively the function and measure in Proposition 5.2, and so

$$
u_{b}-\varphi^{*}=\int_{[0,+\infty)}(\lambda-\varphi(\lambda)) d \tau_{(x, t)}(\lambda)=\int_{[0,+\infty)} h(\lambda) d \tau_{(x, t)}(\lambda)=h^{*}(x, t) \quad \text { a.e. }(x, t) \in Q \text {. }
$$

Moreover, we obtain

$$
\begin{aligned}
\lim _{k \rightarrow \infty} \int_{0}^{T} \xi(t) U_{h, k}^{\rho}(t) d t & =\lim _{k \rightarrow \infty} \int_{Q} h\left(u_{n_{k}}\right) \rho(x) \xi(t) d x d t \\
& =\int_{Q} h^{*}(x, t) \rho(x) \xi(t) d x d t+\langle\mu, \rho \xi\rangle_{Q} \\
& =\int_{0}^{T} \xi(t)\left(\int_{\Omega} h^{*}(x, t) \rho(x) d x+\langle\mu(\cdot, t), \rho\rangle_{\Omega}\right) d t
\end{aligned}
$$

whence by (5.19) there holds

$$
U_{h}^{\rho}(t)=\int_{\Omega} h^{*}(x, t) \rho(x) d x+\langle\mu(\cdot, t), \rho\rangle_{\Omega}
$$

for every $t \in(0, T) \backslash \mathcal{N}$.

Step 2: Assume that, for every $\rho \in C_{c}^{2}(\Omega)$, set

$$
U_{k}^{\rho}(t)=\int_{\Omega} u_{n_{k}}(x, t) \rho(x) d x \quad \text { a.e. } t \in(0, T) .
$$

For $h$ given in (5.20), we infer that

$$
U_{k}^{\rho}(t)=U_{h, k}^{\rho}(t)+U_{\varphi, k}^{\rho}(t)
$$

where $U_{h, k}^{\rho}$ and $U_{\varphi, k}^{\rho}$ are defined in (5.11) and

$$
U_{\varphi, k}^{\rho}(t):=\int_{\Omega} \varphi\left(u_{n_{k}}\right)(x, t) \rho(x) d x \quad \text { a.e. } t \in(0, T)
$$

Based on Proposition 5.2 with (5.25), we will show the following convergence:

$$
U_{k}^{\rho} \rightarrow U^{\rho} \quad \text { in } L^{1}(0, T)
$$

where

$$
U^{\rho}(t)=\int_{\Omega}\left(h^{*}(x, t)+\varphi^{*}(x, t)\right) \rho(x) d x+\langle\mu(\cdot, t), \rho\rangle_{\Omega} .
$$

From (5.20), there holds

$$
h^{*}(x, t)+\varphi^{*}(x, t)=\int_{[0,+\infty)}(h(\lambda)+\varphi(\lambda)) d \tau_{(x, t)}(\lambda)=\int_{[0,+\infty)} \lambda d \tau_{(x, t)}(\lambda)=u_{b}(x, t)
$$

for a.e. $(x, t) \in Q$. 
To prove (5.27), we consider for every $\rho \in C_{c}(\Omega)$ and $\left\{\rho_{k}\right\} \subseteq C_{c}^{2}(\Omega)$ be any sequence such that $\rho_{k} \rightarrow \rho$ uniformly in $\bar{\Omega}$, then we get

$$
\begin{aligned}
\int_{0}^{T}\left|U_{k}^{\rho}(t)-U^{\rho}(t)\right| d t \leq & \int_{0}^{T}\left|U_{k}^{\rho}(t)-U_{k}^{\rho_{j}}(t)\right| d t+\int_{0}^{T}\left|U_{k}^{\rho_{j}}(t)-U^{\rho_{j}}(t)\right| d t \\
& +\int_{0}^{T}\left|U^{\rho_{j}}(t)-U^{\rho}(t)\right| d t
\end{aligned}
$$

By Step 1, one has

$$
\int_{0}^{T}\left|U^{\rho_{j}}(t)-U^{\rho}(t)\right| d t \leq\left\|\rho-\rho_{j}\right\|_{C(\bar{\Omega})} \int_{0}^{T} \mu(\cdot, t)(\Omega) d t+\left\|\rho-\rho_{j}\right\|_{C(\bar{\Omega})} \int_{Q} u_{b}(x, t) d x d t .
$$

Therefore, we obtain

$$
\begin{aligned}
& \int_{0}^{T}\left|U_{k}^{\rho}(t)-U^{\rho}(t)\right| d t \\
& \quad \leq\left\|\rho-\rho_{j}\right\|_{C(\bar{\Omega})} \int_{Q} u_{n_{k}}(x, t) d x d t+\left\|\rho-\rho_{j}\right\|_{C(\bar{\Omega})} \int_{0}^{T} \mu(\cdot, t)(\Omega) d t \\
& \quad+\int_{0}^{T}\left|U_{k}^{\rho_{j}}(t)-U^{\rho_{j}}(t)\right| d t+\left\|\rho-\rho_{j}\right\|_{C(\bar{\Omega})} \int_{Q} u_{b}(x, t) d x d t .
\end{aligned}
$$

By (4.5) and (5.28), one has

$$
\begin{aligned}
\limsup _{k \rightarrow \infty} \int_{0}^{T}\left|U_{k}^{\rho}(t)-U^{\rho}(t)\right| d t \leq & C\left\|\rho-\rho_{j}\right\|_{C(\bar{\Omega})}+\left\|\rho-\rho_{j}\right\|_{C(\bar{\Omega})} \int_{Q} u_{b}(x, t) d x d t \\
& +\left\|\rho-\rho_{j}\right\|_{C(\bar{\Omega})} \int_{0}^{T} \mu(\cdot, t)(\Omega) d t .
\end{aligned}
$$

By letting $j \rightarrow \infty$ in the above inequality, assertion (5.27) holds true. Therefore, for every $\rho$, there exist a subsequence denoted again by $\left\{u_{n_{k}}\right\}$ and a zero Lebesgue measure $\operatorname{set} \mathcal{N} \subset$ $(0, T)$ such that we get

$$
\lim _{k \rightarrow \infty} \int_{\Omega} u_{n_{k}}(t) \rho(x) d t=\int_{\Omega} u_{b}(x, t) \rho(x) d x+\langle\mu(\cdot, t), \rho\rangle_{\Omega}
$$

for any $t \in(0, T) \backslash \mathcal{N}$ and every $\rho \in C_{c}(\Omega)$, hence (5.10) and (5.11).

Proposition 5.4 Suppose that (1.1), (1.4), (R), and (G) hold. Let $\mu$ be given by Proposition 5.2. Then there holds

$$
\psi\left(u_{n_{k}}\right) \stackrel{*}{\rightarrow} \psi^{*}+\alpha \mu \quad \operatorname{in} \mathcal{M}^{+}(Q),
$$

and

$$
f\left(u_{n_{k}}\right) \stackrel{*}{\rightarrow} f^{*}+f^{\prime}(+\infty) \mu \quad \text { in } \mathcal{M}^{+}(Q)
$$


where $f^{*}, \psi^{*} \in L^{\infty}\left((0, T), L^{1}(\Omega)\right)$ is defined by

$$
\psi^{*}(x, t)=\int_{[0,+\infty)} \psi(\lambda) d \tau_{(x, t)}(\lambda) \quad \text { and } \quad f^{*}(x, t)=\int_{[0,+\infty)} f(\lambda) d \tau_{(x, t)}(\lambda)
$$

for a.e. in $Q$.

The proof of Proposition 5.4 is argued as in [14, Proposition 5.2] or [1, Lemma 8.3], for this reason we omit this proof.

Proposition 5.5 Assume that (1.1), (1.4), $(R)$, and $(G)$ hold. Let $\mu$ be given by Proposition 5.2. Then there exist a zero Lebesgue measure set $\mathcal{N} \subset(0, T)$ and a subsequence of $\left\{u_{n_{k}}\right\}$ such that, for any $t \in(0, T) \backslash \mathcal{N}$, there holds

$$
\psi\left(u_{n_{k}}\right) \stackrel{*}{\rightarrow} \psi^{*}(\cdot, t)+\alpha \mu(\cdot, t) \quad \text { in } \mathcal{M}^{+}(\Omega)
$$

and

$$
f\left(u_{n_{k}}\right) \stackrel{*}{\rightarrow} f^{*}(\cdot, t)+f^{\prime}(+\infty) \mu(\cdot, t) \quad \text { in } \mathcal{M}^{+}(\Omega)
$$

Proof Let $\tau \in \mathcal{Y}(Q, \mathbb{R})$ and $\left\{u_{n_{k}}\right\}$ be respectively the Young measure and the subsequence associated with the sequence of Young measure $\left\{\tau^{n}\right\}$. For every $\rho \in C_{c}(\bar{\Omega})$,

$$
G_{k}^{\rho}(t)=\int_{\Omega} \psi\left(u_{n_{k}}\right)(x, t) \rho(x) d x, \quad \text { a.e. } t \in(0, T) .
$$

To prove convergence (5.32), it is enough to show that

$$
\lim _{k \rightarrow+\infty} \int_{0}^{T} G_{k}^{\rho}(t) \xi(t) d t=\int_{0}^{T} G^{\rho}(t) \xi(t) d t
$$

for every $\xi \in C_{c}^{1}(0, T)$, with

$$
\left.G^{\rho}(t)=\int_{\Omega} \psi^{*}(x, t)\right) \rho(x) d x+\alpha \int_{\Omega} \mu(\cdot, t) \rho(x) d x
$$

and $\mu(\cdot, t) \in \mathcal{M}^{+}(\Omega)$ and $\psi^{*} \in L^{\infty}(Q)$ given by (3.4). From assumption (G) and (1.1), the function $\psi \in C^{2}\left(\mathbb{R}_{+}\right)$, and for any $\rho \in C_{c}^{1}(\Omega)$, for every $k \in \mathbb{N}$, there holds

$$
\left\|G_{k}^{\rho}\right\|_{L^{\infty}(0, T)} \leq C\left\|\psi\left(u_{n_{k}}\right)\right\|_{L^{1}(Q)}\|\rho\|_{L^{\infty}(\Omega)} .
$$

Moreover, let us consider $\rho \psi^{\prime}\left(u_{n_{k}}\right) \xi$. For every $\xi \in C_{c}^{1}(0, T)$ as a test function in $\left(P_{n}\right)$, there holds

$$
\begin{aligned}
\int_{0}^{T}\left[G_{k}^{\rho}(t)\right]_{t} \xi d x d t= & -\int_{0}^{T} \xi(t)\left(\int_{\Omega}\left[\psi\left(u_{n_{k}}\right)\right]_{x}\left[\rho(x) \psi^{\prime}\left(u_{n_{k}}\right)\right]_{x} d x\right) d t \\
& +\int_{0}^{T} \xi(t)\left(\int_{\Omega} f\left(u_{n_{k}}\right) \psi^{\prime}\left(u_{n_{k}}\right) \rho(x) d x\right) d t
\end{aligned}
$$


which leads to the fact that the weak derivation of $G_{k}^{\rho} \in W^{1,1}(0, T)$ is such that

$$
\frac{d}{d t} G_{k}^{\rho}(t)=-\int_{\Omega}\left[\psi\left(u_{n_{k}}\right)\right]_{x}\left[\rho(x) \psi^{\prime}\left(u_{n_{k}}\right)\right]_{x} d x+\int_{\Omega} f\left(u_{n_{k}}\right) \psi^{\prime}\left(u_{n_{k}}\right) \rho(x) d x
$$

Since $f, f^{\prime}, \psi^{\prime}$, and $\psi^{\prime \prime}$ are continuous and uniformly bounded (see assumptions (G) and $(R))$, then we have

$$
\begin{aligned}
\int_{0}^{T}\left|\frac{d}{d t} G_{k}^{\rho}(t)\right| d t \leq & \int_{0}^{T}\left|\int_{\Omega}\left[\psi\left(u_{n_{k}}\right)\right]_{x}\left[\rho(x) \psi^{\prime}\left(u_{n_{k}}\right)\right]_{x} d x\right| d t \\
& +\int_{0}^{T}\left|\int_{\Omega} f\left(u_{n_{k}}\right) \psi^{\prime}\left(u_{n_{k}}\right) \rho(x) d x\right| d t .
\end{aligned}
$$

By (4.8), (4.9), (4.11), and assumptions (G) and (R), we may estimate each term of (5.39) as follows:

$$
\begin{aligned}
\int_{0}^{T}\left|\int_{\Omega}\left[\psi\left(u_{n_{k}}\right)\right]_{x}\left[\rho(x) \psi^{\prime}\left(u_{n_{k}}\right)\right]_{x} d x\right| d t \leq & \int_{Q}\left|\left[\psi\left(u_{n_{k}}\right)\right]_{x}\right|\left|\psi^{\prime}\left(u_{n_{k}}\right)\right|\left|\rho^{\prime}(x)\right| d x d t \\
& +\int_{Q}\left|\left[\psi\left(u_{n_{k}}\right)\right]_{x}\right|^{2} \frac{\psi^{\prime \prime}\left(u_{n_{k}}\right)}{\psi^{\prime}\left(u_{n_{k}}\right)} d x d t .
\end{aligned}
$$

Therefore, there exists a positive constant $C_{\rho}=C(\rho)$ such that

$$
\int_{0}^{T}\left|\int_{\Omega}\left[\psi\left(u_{n_{k}}\right)\right]_{x}\left[\rho(x) \psi^{\prime}\left(u_{n_{k}}\right)\right]_{x} d x\right| d t \leq C_{\rho}
$$

On the other hand, we have

$$
\int_{0}^{T}\left|\int_{\Omega} f\left(u_{n_{k}}\right) \psi^{\prime}\left(u_{n_{k}}\right) \rho(x) d x\right| d t \leq L\|\rho\|_{L^{\infty}(\Omega)}\left\|\psi^{\prime}\left(u_{n_{k}}\right)\right\|_{L^{\infty}\left(\mathbb{R}_{+}\right)} \int_{Q} u_{n_{k}} d x d t .
$$

Accordingly, there exists a positive constant $\widetilde{C}_{\rho}=\widetilde{C}(\rho)$ such that

$$
\int_{0}^{T}\left|\int_{\Omega} f\left(u_{n_{k}}\right) \psi^{\prime}\left(u_{n_{k}}\right) \rho(x) d x\right| d t \leq \widetilde{C}_{\rho}
$$

In view of (5.39)-(5.42), the sequence $\left\{G_{h, k}^{\rho}\right\}$ is uniformly bounded in $W^{1,1}(0, T)$, whence relatively compact in $L^{1}(0, T)$. To achieve this proof, we consider for every $\rho \in C_{c}(\Omega)$ and $\left\{\rho_{k}\right\} \subseteq C_{c}^{2}(\Omega)$ be any sequence such that $\rho_{k} \rightarrow \rho$ uniformly in $\bar{\Omega}$, then we get

$$
\begin{aligned}
& \int_{0}^{T}\left|G_{k}^{\rho}(t)-G^{\rho}(t)\right| d t \\
& \leq \int_{0}^{T}\left|G_{k}^{\rho}(t)-G_{k}^{\rho_{j}}(t)\right| d t+\int_{0}^{T}\left|G_{k}^{\rho_{j}}(t)-G^{\rho_{j}}(t)\right| d t+\int_{0}^{T}\left|G^{\rho_{j}}(t)-G^{\rho}(t)\right| d t \\
& \leq\left\|\rho-\rho_{j}\right\|_{C(\bar{\Omega})} \int_{Q} \psi\left(u_{n_{k}}\right)(x, t) d x d t \\
& \quad+\alpha\left\|\rho-\rho_{j}\right\|_{C(\bar{\Omega})} \int_{0}^{T} \mu(\cdot, t)(\Omega) d t+\int_{0}^{T}\left|G_{k}^{\rho_{j}}(t)-G^{\rho_{j}}(t)\right| d t .
\end{aligned}
$$


By (4.5) and (5.43), one has

$$
\begin{aligned}
\limsup _{k \rightarrow \infty} \int_{0}^{T}\left|G_{k}^{\rho}(t)-G^{\rho}(t)\right| d t \leq & C\left\|\rho-\rho_{j}\right\|_{C(\bar{\Omega})}+\left\|\rho-\rho_{j}\right\|_{C(\bar{\Omega})} \int_{Q} \psi^{*}(x, t) d x d t \\
& +\alpha\left\|\rho-\rho_{j}\right\|_{C(\bar{\Omega})} \int_{0}^{T} \mu(\cdot, t)(\Omega) d t .
\end{aligned}
$$

By letting $j \rightarrow \infty$ in the above inequality, assertion (5.32) holds true. We use a similar approach to prove convergence (5.33). Hence, we omit the proof of assertion (5.33).

Remark 5.1 Since $\psi$ is given in (1.1), assumption (H) and (5.8) hold, then there exists a subsequence of $\left\{u_{n_{k}}\right\}$ (not relabeled) such that

$$
\psi\left(u_{n_{k}}\right) \stackrel{*}{\rightarrow} \alpha u+\beta \varphi^{*} \quad \text { in } \mathcal{M}^{+}(Q),
$$

where $\varphi^{*}$ is defined by (5.22) and $u$ is given in Proposition 5.2. Similarly, we get

$$
\psi\left(u_{n_{k}}\right) \stackrel{*}{\rightarrow} \alpha u(\cdot, t)+\beta \varphi^{*}(\cdot, t) \quad \text { in } \mathcal{M}^{+}(\Omega)
$$

where $t \in(0, T) \backslash \mathcal{N}$.

Proposition 5.6 Suppose that (1.1), (1.4), (R), and (G) hold. Let (5.8) be the Lebesgue decomposition of $u$. Then there holds

$$
u_{b}=u_{r} \quad \text { a.e. in } Q ; \quad \mu=u_{s} \quad \text { a.e. in } \mathcal{M}^{+}(Q) \text {. }
$$

Moreover,

$$
\psi\left(u_{r}\right)=\int_{[0,+\infty)} \psi(\lambda) d \tau_{(x, t)}(\lambda) \text { and } f\left(u_{r}\right)=\int_{[0,+\infty)} f(\lambda) d \tau_{(x, t)}(\lambda)
$$

where $u_{r} \in L^{\infty}\left((0, T), L^{1}(\Omega)\right)$ is the density of an absolutely continuous part of $u$.

Proof Let us recall the definition of the weak solution to problem $\left(P_{n}\right)$ with $m \in \mathbb{N} \backslash\{0\}$ such that

$$
\int_{Q}\left\{u_{n_{k}}^{m} \xi_{t}+\psi_{m}\left(u_{n_{k}}^{m}\right) \xi_{x x}\right\} d x d t+\int_{Q} f_{m}\left(u_{n_{k}}^{m}\right) \xi d x d t=\left\langle u_{0 n_{k}}^{m}, \xi(\cdot, 0)\right\rangle_{\Omega^{\prime}}
$$

whenever $\psi_{m}(\lambda)=\psi(\lambda) \chi_{\left(-m, \frac{1}{m}\right)}(\lambda)$ and $f_{m}(\lambda)=f(\lambda) \chi_{\left(-m, \frac{1}{m}\right)}(\lambda)$. From Proposition $5.2-$ Proposition 5.4, the following assertions hold true:

$$
\begin{aligned}
& \lim _{n_{k} \rightarrow \infty} \int_{0}^{\bar{t}}\left|\int_{\Omega} u_{n_{k}}^{m} \xi_{t} d x-\int_{\Omega} u_{b}^{m} \xi_{t} d x-\left\langle\mu(\cdot, t), \xi_{t}\right\rangle_{\Omega}\right| d t=0, \\
& \lim _{n_{k} \rightarrow \infty} \int_{0}^{\bar{t}}\left|\int_{\Omega} \psi_{m}\left(u_{n_{k}}^{m}\right) \xi_{x x} d x-\int_{\Omega} \psi_{m}^{*} \xi_{x x} d x-\left\langle\alpha \mu(\cdot, t), \xi_{x x}\right\rangle_{\Omega}\right| d t=0,
\end{aligned}
$$


and

$$
\lim _{n_{k} \rightarrow \infty} \int_{0}^{\bar{t}}\left|\int_{\Omega} f_{m}\left(u_{n_{k}}^{m}\right) \xi d x-\int_{\Omega} f_{m}^{*} \xi d x-\left\langle f^{\prime}(+\infty) \mu(\cdot, t), \xi\right\rangle_{\Omega}\right| d t=0
$$

where

$$
\begin{aligned}
& u_{b}^{m}(x, t)=\int_{[0,+\infty)} \lambda \chi_{\left(-m, \frac{1}{m}\right)}(\lambda) d \tau_{(x, t)}(\lambda), \\
& \psi_{m}^{*}(x, t)=\int_{[0,+\infty)} \psi(\lambda) \chi_{\left(-m, \frac{1}{m}\right)}(\lambda) d \tau_{(x, t)}(\lambda),
\end{aligned}
$$

and

$$
f_{m}^{*}(x, t)=\int_{[0,+\infty)} f(\lambda) \chi_{\left(-m, \frac{1}{m}\right)}(\lambda) d \tau_{(x, t)}(\lambda)
$$

belong to $L^{\infty}\left((0, T), L^{1}(\Omega)\right)$. Since we assume that $\xi$ is a solution to the backward parabolic equations $(v \cdot \alpha)$ such that $\xi_{v} \in C(Q)$, we get

$$
\begin{aligned}
\lim _{n_{k} \rightarrow \infty} & {\left[\int_{\Omega \times(0, \bar{t})}\left\{u_{n_{k}}^{m} \xi_{t}+\psi_{m}\left(u_{n_{k}}^{m}\right) \xi_{x x}\right\} d x d t+\int_{\Omega \times(0, \bar{t})} f_{m}\left(u_{n_{k}}^{m}\right) \xi d x d t\right] } \\
= & \int_{\Omega \times(0, \bar{t})}\left\{u_{b}^{m}(x, t) \xi_{t}(x, t)+\psi_{m}^{*}(x, t) \xi_{x x}(x, t)+f_{m}^{*}(x, t) \xi(x, t)\right\} d x d t \\
& +\int_{0}^{\bar{t}}\left\langle\mu(\cdot, t), \xi_{v}\right\rangle_{\Omega}
\end{aligned}
$$

and

$$
\lim _{n_{k} \rightarrow \infty} \int_{\Omega} u_{0 n_{k}}^{m} \xi(\cdot, 0) d x=\int_{\Omega} u_{0 r} \chi_{\left(-m, \frac{1}{m}\right)}\left(u_{0 r}\right) \xi(\cdot, 0) d x+\int_{\Omega} u_{0 s} \xi(\cdot, 0) d x
$$

Since, for all $u \geq 0,\left|\psi_{m}(u)\right| \leq(\alpha+\beta) u \chi_{\left(-m, \frac{1}{m}\right)}(u)$ and $\left|f_{m}(u)\right| \leq L u \chi_{\left(-m, \frac{1}{m}\right)}(u)$, then $\left|\psi_{m}^{*}(x, t)\right| \leq(\alpha+\beta) u_{b}^{m}$, where $L:=\left\|f^{\prime}(u)\right\|_{L^{\infty}\left(\mathbb{R}_{+}\right)}$and $\left|f_{m}^{*}(x, t)\right| \leq L u_{b}^{m}$. Therefore, the following convergence holds: $u_{b}^{m} \rightarrow 0, \psi_{m}^{*} \rightarrow 0$, and $f_{m}^{*} \rightarrow 0$ a.e. in $Q$ as $m \rightarrow \infty$. For all $\bar{t} \in(0, T) \backslash \mathcal{N}$, with $|\mathcal{N}|=0$. From (5.48)-(5.53), we deduce that

$$
\langle\mu(\cdot, \bar{t}), \xi\rangle_{\Omega}=\int_{0}^{\bar{t}}\left\langle\mu(\cdot, \bar{t}), \xi_{v}\right\rangle_{\Omega} d t+\left\langle u_{0 s}, \xi(\cdot, 0)\right\rangle_{\Omega}
$$

Taking the test function $\xi \in C\left([0, T], C_{0}^{1}(\Omega)\right)$ such that $\xi_{v}=0$ in $\Omega \times(0, \bar{t})$. Hence $\mu(\cdot, t)$ is singular with respect to the Lebesgue measure and $\mu(\cdot, t)=[\mu(\cdot, t)]_{s}=u_{s}(\cdot, \bar{t})$ for any $\bar{t} \in(0, T) \backslash \mathcal{N}$.

\section{Characterization of the limit Young measure}

The main result of this section is given by the following proposition.

Proposition 6.1 Let $v \in L^{2}\left((0, T), H_{0}^{1}(\Omega)\right) \cap L^{\infty}\left((0, T), H_{0}^{1}(\Omega)\right) \cap L^{\infty}(Q)$ be the limit offunction given in Proposition 5.1. (i) Let $\left\{\sigma_{n}\right\} \subseteq \mathcal{Y}(Q, \mathbb{R})$ be the sequence of Young measures on 
$Q \times \mathbb{R}$ associated with $\left\{\psi\left(u_{n}\right)\right\}$. Then there exist Young measure $\sigma \in \mathcal{Y}(Q, \mathbb{R})$ and a subsequence $\left\{\psi\left(u_{n_{k}}\right)\right\} \subseteq\left\{\psi\left(u_{n}\right)\right\}$ such that

$$
\sigma_{n_{k}} \rightarrow \sigma \text { narrowly on } Q \times \mathbb{R}
$$

for almost everywhere $(x, t) \in Q$, there holds

$$
\sigma_{(x, t)}=\delta_{\{v(x, t)\}},
$$

where $\sigma_{(x, t)} \in \mathcal{P}(\mathbb{R})$ is the disintegration of $\sigma$.

(ii) Let $\left\{\tau_{n}\right\} \subseteq \mathcal{Y}(Q, \mathbb{R})$ be the sequence of Young measures on $Q \times \mathbb{R}$ associated with $\left\{u_{n}\right\}$. Then there exist Young measure $\tau \in \mathcal{Y}(Q, \mathbb{R})$ and a subsequence $\left\{u_{n_{k}}\right\} \subseteq\left\{u_{n}\right\}$ such that

$$
\tau_{n_{k}} \rightarrow \tau \text { narrowly on } Q \times \mathbb{R}
$$

Denoting by $\tau_{(x, t)}$ the disintegration of $\tau$, for almost everywhere $(x, t) \in Q$, there hold

$$
\tau_{(x, t)}=\delta_{\psi^{-1}(v(x, t))} \text { and } \operatorname{supp} \tau_{(x, t)} \subseteq[0,+\infty) \text {, }
$$

where $\delta_{\left\{\psi^{-1}(v(x, t))\right\}}$ is a Dirac mass concentration at $\psi^{-1}(v(x, t))$.

The proof of Proposition 6.1 is postponed now, and it will be made at the end of this section due to a number of intermediate steps: Proposition 6.2, Proposition 6.3, and Proposition 6.4.

Let us consider the function $V$ as follows:

$$
V(s)= \begin{cases}s & \text { if } s>0 \\ 0 & \text { if } s \leq 0\end{cases}
$$

Proposition 6.2 Let $V$ be the function (6.5), and let $\tau_{(x, t)}$ be the disintegration of the limit Young measure $\tau$ mentioned in Proposition 5.2. Then there exist a subsequence $\left\{u_{n_{k}}\right\} \subseteq\left\{u_{n}\right\}$ and a zero Lebesgue measure set $\mathcal{N} \subset(0, T)$ such that

$$
V\left(u_{n_{k}}\right)(\cdot, t) \rightarrow \int_{[0,+\infty)} V(\lambda) d \tau_{(\cdot, t)}(\lambda) \quad \text { in } L^{1}(\Omega)
$$

for every $t \in(0, T) \backslash \mathcal{N}$.

Proof Fix any $\rho \in C_{c}^{1}(\Omega)$ and $\epsilon>0$. Set

$$
W_{\epsilon, n}^{\rho}(t)=\int_{\Omega} V_{\epsilon}\left(u_{n}\right)(x, t) \rho(x) d x \quad(\text { a.e. } t \in(0, T)),
$$

where

$$
V_{\epsilon}(s)=\int_{0}^{s} \mathcal{T}_{\epsilon}(\sigma) d \sigma \quad \text { and } \quad \mathcal{T}_{\epsilon}(\sigma)=1 \quad \text { if } \sigma>\frac{1}{\epsilon} ; \quad \mathcal{T}_{\epsilon}(\sigma)=\epsilon \sigma \quad \text { if } \sigma \leq \frac{1}{\epsilon} .
$$

Let us first show that $W_{\epsilon, n}^{\rho} \in W^{1,1}(0, T)$. 
By [28, Proposition 4.10], there exists an open set $\Omega_{n}^{t} \subseteq \Omega$ (depending on $t$ ) such that $\operatorname{dist}\left(\Omega_{n}^{t}, S^{t}\right)>0$ and $\operatorname{supp} \mathcal{T}_{\epsilon}\left(u_{n}\right)(\cdot, t) \subset \Omega_{n}^{t}$ and $u_{n}(\cdot, t) \in H^{1}\left(\Omega_{n}^{t}\right)$, where $S^{t}=\{x \in$ $\left.\bar{\Omega} / \psi\left(u_{r}\right)(x, t) \neq \infty\right\}$, then there holds

$$
u_{n t}(\cdot, t)=\left[\psi\left(u_{n}\right)(\cdot, t)\right]_{x x}+f\left(u_{n}\right)(\cdot, t) \quad \text { a.e. in } \Omega_{n}^{t} .
$$

The weak derivative of $W_{\epsilon, n}^{\rho}$ is given by

$$
\begin{aligned}
\frac{d}{d t} W_{\epsilon, n}^{\rho}(t)= & \int_{\Omega}\left|\left[\psi\left(u_{n}\right)\right]_{x}\right|^{2} \frac{\mathcal{T}_{\epsilon}^{\prime}\left(u_{n}\right)}{\psi^{\prime}\left(u_{n}\right)} \rho(x) d x+\int_{\Omega}\left[\psi\left(u_{n}\right)\right]_{x} \mathcal{T}_{\epsilon}\left(u_{n}\right) \rho^{\prime}(x) d x \\
& +\int_{\Omega} f\left(u_{n}\right) \mathcal{T}_{\epsilon}\left(u_{n}\right) \rho(x) d x
\end{aligned}
$$

for every $t \in(0, T) \backslash \mathcal{N}$.

$$
\begin{aligned}
\int_{0}^{T} \mid & \frac{d}{d t} W_{\epsilon, n}^{\rho}(t) \mid d t \\
\leq & \left.\int_{0}^{T}\left|\int_{\Omega}\right|\left[\psi\left(u_{n}\right)\right]_{x}\right|^{2} \frac{\mathcal{T}_{\epsilon}^{\prime}\left(u_{n}\right)}{\psi^{\prime}\left(u_{n}\right)} \rho(x) d x\left|d t+\int_{0}^{T}\right| \int_{\Omega}\left[\psi\left(u_{n}\right)\right]_{x} \mathcal{T}_{\epsilon}\left(u_{n}\right) \rho^{\prime}(x) d x \mid d t \\
& \quad+\int_{0}^{T}\left|\int_{\Omega} f\left(u_{n}\right) \mathcal{T}_{\epsilon}\left(u_{n}\right) \rho(x) d x\right| d t .
\end{aligned}
$$

In view of (1.1), (4.5), (4.8), (6.8), and assumption (G), there exists a positive constant $\widehat{C}_{\rho}=$ $\widehat{C}(\rho)$ such that

$$
\begin{aligned}
& \int_{0}^{T}\left|\frac{d}{d t} W_{\epsilon, n}^{\rho}(t)\right| d t \\
& \leq\left\|\frac{\mathcal{T}_{\epsilon}^{\prime}\left(u_{n}\right)}{\psi^{\prime}\left(u_{n}\right)}\right\|_{L^{\infty}\left(\mathbb{R}_{+}\right)}\|\rho\|_{C(\bar{\Omega})} \int_{Q}\left|\left[\psi\left(u_{n}\right)\right]_{x}\right|^{2} d x d t \\
& \quad+\left\|\mathcal{T}_{\epsilon}^{\prime}\left(u_{n}\right)\right\|_{L^{\infty}\left(\mathbb{R}_{+}\right)}\left\|\rho^{\prime}\right\|_{C(\bar{\Omega})} \int_{Q}\left|\left[\psi\left(u_{n}\right)\right]_{x}\right| d x d t+C\|\rho\|_{C(\bar{\Omega})}:=\widehat{C}(\rho) .
\end{aligned}
$$

Notice that estimate (6.12) holds for every $\alpha \neq 0$.

Then sequence $\left\{V_{\epsilon}\left(u_{n}\right)\right\}$ is uniformly bounded in $L^{1}(\Omega)$ and equi-integrable. In fact, for almost everywhere $(x, t) \in Q$, there holds

$$
\int_{\Omega}\left|V_{\epsilon}\left(u_{n}\right)\right| d x=\int_{\Omega}\left|\int_{0}^{u_{n}(x, t)} \mathcal{T}_{\epsilon}(\sigma) d \sigma\right| d x \leq \int_{\Omega}\left|u_{n}(x, t)\right| d x \leq C\left\|u_{0}\right\|_{\mathcal{M}^{+}(\Omega)} .
$$

By (6.10) and (6.11), the family $\left\{W_{\epsilon, n}^{\rho}(t)\right\}$ is uniformly bounded in $W^{1,1}(0, T)$. Therefore, there exist a subsequence $\left\{u_{n_{k}}\right\} \subseteq\left\{u_{n}\right\}$ and a function $W_{\epsilon}^{\rho} \in L^{1}(0, T)$ such that

$$
W_{\epsilon, n_{k}}^{\rho} \rightarrow W_{\epsilon}^{\rho} \quad \text { in } L^{1}(0, T)
$$

where

$$
W_{\epsilon}^{\rho}(t)=\int_{[0,+\infty)} V_{\epsilon}(\lambda) d \tau_{(x, t)}(\lambda)
$$


Notice that the proof of (6.14) is argued as in Proposition 5.3. Therefore, for any $\xi \in$ $L^{\infty}(0, T)$, we have

$$
\begin{aligned}
\lim _{n_{k} \rightarrow \infty} \int_{0}^{T} W_{\epsilon, n_{k}}^{\rho}(t) \xi(t) d t & =\lim _{k \rightarrow \infty} \int_{0}^{T} \xi(t) d t \int_{\Omega} V_{\epsilon}\left(u_{n_{k}}\right) \rho(x) d x \\
& =\lim _{n_{k} \rightarrow \infty} \int_{Q} V_{\epsilon}\left(u_{n_{k}}\right) \rho(x) \xi(t) d x d t \\
& =\int_{Q}\left(\int_{[0,+\infty)} V_{\epsilon}(\lambda) d \tau_{(x, t)}(\lambda)\right) \rho(x) \xi(t) d x d t .
\end{aligned}
$$

By the dominated convergence theorem, there holds

$$
\lim _{\epsilon \rightarrow 0} \lim _{n_{k} \rightarrow \infty} \int_{0}^{T} W_{\epsilon, n_{k}}^{\rho}(t) \xi(t) d t=\int_{0}^{T} \xi(t) d t \int_{\Omega} V^{*}(x, t) \rho(x) d x,
$$

whenever

$$
W^{\rho}(t)=\int_{\Omega} V^{*}(x, t) \rho(x) d x=\int_{\Omega}\left(\int_{[0,+\infty)} V(\lambda) d \tau_{(x, t)}(\lambda)\right) \rho(x) d x
$$

for any $t \in(0, T) \backslash \mathcal{N}$.

Consider the orthogonal basic of $L^{2}(\Omega)$ given by the operator $-\Delta$ with homogeneous Dirichlet conditions. Let $\left\{\mu_{i}\right\}$ be the corresponding sequence of eigenvalues. Let $P_{n}, Q_{n}$ : $L^{2}(\Omega) \rightarrow H_{0}^{1}(\Omega), P_{n}+Q_{n}=I$ be the projection operator defined as follows:

$$
P_{n} \widetilde{f}=\sum_{\mu_{i} \leq n} \widetilde{f}_{i} \eta_{i}, \quad Q_{n} \widetilde{f}=\sum_{\mu_{i} \geq n} \widetilde{f}_{i} \eta_{i}, \quad \text { and } \quad \tilde{f}_{i}=\int_{\Omega} \tilde{f} \eta_{i} d x
$$

for any $\tilde{f} \in L^{2}(\Omega)$.

Proposition 6.3 There exists $C>0$ such that

$$
\left\|P_{n} \psi\left(u_{n}\right)\right\|_{L^{2}\left((0, T), H_{0}^{1}(\Omega)\right)}+n^{\frac{1}{2}}\left\|P_{n} \psi\left(u_{n}\right)\right\|_{L^{2}(Q)} \leq C .
$$

We omit the proof of Proposition 6.3, the reader may refer to [28, Lemma 1], and this proposition is used in the proof of the following proposition.

Proposition 6.4 Let $\left\{u_{n_{k}}\right\}$ be a subsequence mentioned in Proposition 6.2 and $\bar{v}$ be the disintegration of the limit Young measure $\tau$ in Proposition 5.2. Let $V$ be function (6.3), set also $F:=\widetilde{f} \circ \psi$ with $\tilde{f} \in C(\mathbb{R})$ such that $\|\widetilde{f}\|_{L^{\infty}(\mathbb{R})}+\left\|\tilde{f}^{\prime}\right\|_{L^{\infty}(\mathbb{R})} \leq C$ for some constant $C>0$. Then there holds

$$
\int_{[0,+\infty)} F(\lambda) V(\lambda) d \tau_{(x, t)}(\lambda)=\int_{[0,+\infty)} F(\lambda) d \tau_{(x, t)}(\lambda) \cdot \int_{[0,+\infty)} V(\lambda) d \tau_{(x, t)}(\lambda) .
$$

We omit the proof of Proposition 6.4, the reader should refer to [28, Proposition 6] for details. The importance of recalling this Proposition 6.4 is that it can be proved for almost everywhere $(x, t) \in Q$, the disintegration measure $\sigma_{(x, t)}$ is the Dirac mass concentrated at the point $\psi^{*}(x, t)$, where $\psi^{*}(x, t)$ is given by (5.31). 
Now we can prove the main result of this section.

Proof of Proposition 6.1 (i) By Proposition 5.1, the function $v \in L^{2}\left((0, T), H_{0}^{1}(\Omega)\right) \cap$ $L^{\infty}\left((0, T), H_{0}^{1}(\Omega)\right) \cap L^{\infty}(Q)$ is such that (5.1), (5.2), and (5.4) hold. By statement (6.20), we choose the suitable functions $F$ and $V$, then it can be proven for almost everywhere $(x, t) \in Q$, the disintegration $\sigma$ (probably replacing $\sigma_{(x, t)}$ by $\tau_{(x, t)}$ ) is a Dirac mass concentrated at $\psi^{*}(x, t)$ such that

$$
\psi\left(u_{n_{k}}\right) \rightarrow \psi^{*}(x, t)=\int_{[0,+\infty)} \psi(\lambda) d \sigma_{(x, t)}(\lambda) \quad \text { in } L^{1}(Q) .
$$

The relevant proof is similar to that given in [41] and [42, Lemmas 5.1-5.2 and Theorem 2.12], then we omit it. To achieve the proof of the assertion (6.2), we should show that $\psi^{*}=v$ a.e. in $Q$. According to Proposition 5.6 and Proposition 5.2, we obtain

$$
\psi^{*}(x, t)=\int_{[0,+\infty)} \psi(\lambda) d \sigma_{(x, t)}(\lambda)=\psi\left(\int_{[0,+\infty)} \lambda d \sigma_{(x, t)}(\lambda)\right)=\psi\left(u_{r}\right)=v(x, t)
$$

It follows that

$$
\sigma_{(x, t)}=\delta_{\left\{\psi^{*}(x, t)\right\}}=\delta_{\{v(x, t)\}} .
$$

(ii) Convergence (6.3) is a consequence of the Prokhorov theorem, since the sequence $\left\{u_{n}\right\}$ is uniformly bounded in $L^{1}(Q)$ (see (4.5)). Furthermore, there exists a subsequence $\left\{u_{n_{k}}\right\} \subseteq\left\{u_{n}\right\}$ such that (5.1)-(5.4) and (6.2) hold. Since $\psi^{\prime}>0$, then $u_{n_{k}} \rightarrow \psi^{-1}(v(x, t))$ a.e. in $Q$. Hence (6.4) is satisfied.

Remark 6.1 (i) Assume that (1.1) and (G) are satisfied and $v \in L^{2}\left((0, T), H_{0}^{1}(\Omega)\right) \cap$ $L^{\infty}\left((0, T), H_{0}^{1}(\Omega)\right) \cap L^{\infty}(Q)$ as in Proposition 5.1, then $\widetilde{S}=\{(x, t) \in \bar{Q} / v(x, t)=\infty\}$ has a zero Lebesgue measure. Moreover,

$$
u_{r}(x, t)=\psi^{-1}(v(x, t)) \quad \text { a.e. }(x, t) \in \bar{Q} \backslash \widetilde{S} \quad \text { and } \quad \operatorname{supp} u_{s} \subseteq \widetilde{S} .
$$

(ii) $u_{r} \in H^{1}\left(Q_{0}\right)$ for any open subset $Q_{0} \subseteq Q$ such that $\operatorname{dist}\left(\bar{Q}_{0}, \widetilde{S}\right)>0$ and $u_{r} \in C(\bar{Q} \backslash \widetilde{S})$, then

$$
\lim _{\operatorname{dist}(\overline{\mathrm{Q}}, \widetilde{S}) \rightarrow 0} u_{r}(x, t)=0
$$

Proof of Theorem 3.1 The existence of solutions to problem (P) with qualitative properties follows from the statement of Proposition 5.2 - Proposition 5.6 and Proposition 6.1.

\section{Decay estimate of solutions}

To establish the decay estimates, we make use of suitable test functions in the definition of weak solutions to the approximation problem (P) and the lower semi-continuity of the total variation theorem, then the estimate follows. 
Proof of Theorem 3.2 Let us consider the approximation problem of $\left(Z_{n}\right)$ given by

$$
\begin{cases}v_{n t}=\left[\psi\left(v_{n}\right)\right]_{x x}+f\left(u_{n}\right) & \text { in } Q:=\Omega \times(0, T), \\ v_{n}=0 & \text { on } \partial \Omega \times(0, T), \\ v_{n}(x, 0)=0 & \text { in } \Omega .\end{cases}
$$

For every $\epsilon>0$, we consider that $\left\{\eta_{\epsilon}(t)\right\}$ is a sequence of smooth functions such that $\left\|\eta_{\epsilon}(t)\right\|_{L^{1}(0, T)} \leq C$ and $\eta_{\epsilon}(t) \stackrel{*}{\rightarrow} \delta_{t}$ in $\mathcal{M}^{+}(0, T)$. Let us choose $\xi(x, t)=\operatorname{sign}\left(u_{n}(x, t)-\right.$ $\left.v_{n}(x, t)\right) \int_{t}^{T} \eta_{\epsilon}(s) s^{\alpha} d s$ as a test function in the approximation problem $\left(P_{n}\right)-\left(Z_{n}\right)$, then we have

$$
\begin{aligned}
& \int_{\Omega} \int_{0}^{T}\left|u_{n}(x, t)-v_{n}(x, t)\right| \eta_{\epsilon}(t) t^{\alpha} d x d t \\
& =\int_{\Omega} \int_{0}^{T} u_{0 n}(x) \eta_{\epsilon}(t) t^{\alpha} d x d t \\
& \quad+\int_{\Omega} \int_{0}^{T}\left[\psi\left(u_{n}\right)-\psi\left(v_{n}\right)\right]_{x x} \operatorname{sign}\left(u_{n}(x, t)-v_{n}(x, t)\right) \int_{t}^{T} \eta_{\epsilon}(s) s^{\alpha} d s d x d t .
\end{aligned}
$$

By the integration by parts, the second term on the right-hand side vanishes, and we estimate the first term on the right-hand side, then we obtain

$$
\int_{\Omega} \int_{0}^{T}\left|u_{n}(x, t)-v_{n}(x, t)\right| \eta_{\epsilon}(t) t^{\alpha} d x d t \leq C\left\|u_{0}\right\|_{\mathcal{M}^{+}(\Omega)}
$$

where $C=C(T)>0$. By letting $\epsilon \rightarrow 0^{+}$, we deduce that

$$
t^{\alpha} \int_{\Omega}\left|u_{n}(x, t)-v_{n}(x, t)\right| d x \leq C\left\|u_{0}\right\|_{\mathcal{M}^{+}(\Omega)}
$$

By [9, Theorem 1, Sect. 5.2.1], the lower semi-continuity of the total variation, we get

$$
t^{\alpha}\|u(\cdot, t)-v(\cdot, t)\|_{\mathcal{M}^{+}(\Omega)} \leq \liminf _{n \rightarrow+\infty} t^{\alpha} \int_{\Omega}\left|u_{n}(x, t)-v_{n}(x, t)\right| d x \leq C\left\|u_{0}\right\|_{\mathcal{M}^{+}(\Omega)}
$$

for a.e. $t \in(0, T)$ and $\alpha>0$. Estimates (3.5) and (3.6) are achieved.

Proof of Theorem 3.3 We argue this proof in two steps:

Step 1. We show that the solution of the nonlinear elliptic equation $(S)$ is Radon measurevalued.

To prove that the pseudo-stationary solutions $w$ are Radon measure-valued solutions, we consider the approximation problem

$$
\begin{cases}-\left[\psi\left(w_{n}\right)\right]_{x x}+w_{n}=u_{0 n} & \text { in } \Omega, \\ w_{n}=0 & \text { on } \partial \Omega, \\ w_{n}(x) \geq 0 & \text { in } \Omega,\end{cases}
$$


where $\left\{w_{n}\right\} \subseteq H_{0}^{1}(\Omega) \cap L^{\infty}(\Omega)$ and $\left\{u_{0 n}\right\} \subseteq C_{0}^{\infty}(\Omega)$ satisfies hypothesis (4.2). Let us consider the function $\mathcal{T}_{\epsilon}$ for any $\epsilon>0$ defined above such that

$$
\mathcal{T}_{\epsilon}(s)= \begin{cases}1 & \text { if } s>\frac{1}{\epsilon} \\ \epsilon S & \text { if } s \leq \frac{1}{\epsilon}\end{cases}
$$

Notice that $0 \leq \mathcal{T}_{\epsilon}(s) \leq 1$ in $\mathbb{R}_{+}, \mathcal{T}_{\epsilon}(s) \rightarrow 1$ as $\epsilon \rightarrow 0^{+}$. Taking $\mathcal{T}_{\epsilon}\left(w_{n}\right)$ as a function in $\left(S_{n}\right)$, we obtain

$$
\int_{\Omega} \psi^{\prime}\left(w_{n}\right) \mathcal{T}_{\epsilon}^{\prime}\left(w_{n}\right)\left|w_{n x}\right|^{2} d x+\int_{\Omega} \mathcal{T}_{\epsilon}\left(w_{n}\right) w_{n} d x \leq\left\|u_{0}\right\|_{\mathcal{M}^{+}(\Omega)} .
$$

By assumption (G), there holds

$$
\left\|w_{n}\right\|_{L^{1}(\Omega)} \leq\left\|u_{0}\right\|_{\mathcal{M}^{+}(\Omega)}
$$

On the other hand, we consider $T_{K}\left(\psi\left(w_{n}\right)\right)=\left(1+\frac{1}{2} g_{K}\left(\psi\left(w_{n}\right)\right)\right) \psi\left(w_{n}\right) \chi_{(1,1+\epsilon)}\left(\psi\left(w_{n}\right)\right)$ (see the proof of Proposition 4.2) is a test function in the approximation problem $\left(S_{n}\right)$, then we obtain

$$
\int_{\Omega}\left|\left[\psi\left(w_{n}\right)\right]_{x}\right|^{2} T_{K}^{\prime}\left(\psi\left(w_{n}\right)\right) d x+\int_{\Omega} w_{n} T_{K}\left(\psi\left(w_{n}\right)\right) d x=\int_{\Omega} u_{0 n} T_{K}\left(\psi\left(w_{n}\right)\right) d x .
$$

Since $1 \leq T_{K}^{\prime}\left(\psi\left(w_{n}\right)\right) \leq 2$ and $T_{K}\left(\psi\left(w_{n}\right)\right) \in L^{\infty}\left(\mathbb{R}_{+}\right)$, for every $K$, there holds

$$
\int_{\Omega}\left|\left[\psi\left(w_{n}\right)\right]_{x}\right|^{2} d x+\int_{\Omega} w_{n} T_{K}\left(\psi\left(w_{n}\right)\right) d x \leq\left\|T_{K}\left(\psi\left(w_{n}\right)\right)\right\|_{L^{\infty}\left(\mathbb{R}_{+}\right)} \int_{\Omega} u_{0 n} d x .
$$

Since $w_{n} \geq 0$ in $\Omega$ and assumption (4.2) holds, then we get

$$
\left\|\psi\left(w_{n}\right)\right\|_{H_{0}^{1}(\Omega)} \leq C\left\|u_{0}\right\|_{\mathcal{M}^{+}(\Omega)} .
$$

As argued in [18] and estimates (7.3) and (7.4), problem $(S)$ admits a Radon measurevalued solution i.e. $w \in \mathcal{M}^{+}(\Omega)$.

Step 2. We show that convergence (3.8) holds true.

Let us consider $\xi(x, t)=\operatorname{sign}\left(u_{n}(x, t)-w_{n}(x)\right) \int_{t}^{T} \eta_{\epsilon}(s) s^{\alpha} d s$ as a test function in the approximation problems $\left(S_{n}\right)$ and $\left(P_{n}\right)$, then we have

$$
\begin{aligned}
& \int_{\Omega} \int_{0}^{T}\left|u_{n}(x, t)-w_{n}(x)\right| \eta_{\epsilon}(t) t^{\alpha} d x d t \\
& =\int_{\Omega} \int_{0}^{T}\left|u_{0 n}(x)-w_{n}(x)\right| \eta_{\epsilon}(t) t^{\alpha} d x d t \\
& \quad+\int_{\Omega} \int_{0}^{T}\left[\psi\left(u_{n}\right)-\psi\left(w_{n}\right)\right]_{x x} \operatorname{sign}\left(u_{n}(x, t)-w_{n}(x)\right) \int_{t}^{T} \eta_{\epsilon}(s) s^{\alpha} d s d x d t \\
& \left.\quad+\int_{\Omega} \int_{0}^{T}\left[f\left(u_{n}\right)+w_{n}\right)\right] \operatorname{sign}\left(u_{n}(x, t)-w_{n}(x)\right) \int_{t}^{T} \eta_{\epsilon}(s) s^{\alpha} d s d x d t \\
& \quad-\int_{\Omega} \int_{0}^{T} u_{0 n} \operatorname{sign}\left(u_{n}(x, t)-w_{n}(x)\right) \int_{t}^{T} \eta_{\epsilon}(s) s^{\alpha} d s d x d t .
\end{aligned}
$$


By the integration by parts, the second term on the right-hand side of the above equations vanishes, and we make use of estimate (7.3) and assumption (R). Then we can deduce that

$$
\int_{\Omega} \int_{0}^{T}\left|u_{n}(x, t)-w_{n}(x)\right| \eta_{\epsilon}(t) t^{\alpha} d x d t \leq C\left\|u_{0}\right\|_{\mathcal{M}^{+}(\Omega)} .
$$

By letting $\epsilon \rightarrow 0^{+}$, therefore we obtain

$$
t^{\alpha} \int_{\Omega}\left|u_{n}(x, t)-w_{n}(x)\right| d x \leq C\left\|u_{0}\right\|_{\mathcal{M}^{+}(\Omega)},
$$

where $C=C(T)>0$. By virtue of [9, Theorem, Sect. 5.2.1], the lower semi-continuity of the total variation, we infer that

$$
t^{\alpha}\|u(\cdot, t)-w(\cdot)\|_{\mathcal{M}^{+}(\Omega)} \leq \liminf _{n \rightarrow+\infty} t^{\alpha} \int_{\Omega}\left|u_{n}(x, t)-w_{n}(x)\right| d x \leq C(T)\left\|u_{0}\right\|_{\mathcal{M}^{+}(\Omega)}
$$

for a.e. $t \in(0, T)$ and $\alpha>0$. Since the constant $C$ in estimate (7.6) is also uniform $T$, then by passing to the limit as $t \rightarrow+\infty$ in the following inequality

$$
\|u(\cdot, t)-w(\cdot)\|_{\mathcal{M}^{+}(\Omega)} \leq C(T) \frac{\left\|u_{0}\right\|_{\mathcal{M}^{+}(\Omega)}}{t^{\alpha}},
$$

convergence (3.8) is achieved.

\section{Acknowledgements}

The authors are available for reviewers' comments and suggestions.

Funding

This work was partially supported by the National Natural Sciences Foundation of China (No: 11571057).

Availability of data and materials

Not applicable.

\section{Declarations}

\section{Consent for publication}

All authors read and approved the final version of the manuscript.

\section{Competing interests}

The authors declare that they have no competing interests.

\section{Authors' contributions}

All authors contributed equally. All authors read and approved the final manuscript.

\section{Publisher's Note}

Springer Nature remains neutral with regard to jurisdictional claims in published maps and institutional affiliations.

Received: 22 June 2021 Accepted: 2 November 2021 Published online: 27 November 2021

\section{References}

1. Marco, P., Michaela, P.M., Flavia, S.: Existence of solutions to a class of weakly coercive diffusion equations with singular initial data. Advances in Differential Equations 22(11-12), 893-963 (2017)

2. Feireisl, E., Frédérique, S.: Convergence for degenerate parabolic equations. J. Differential Equations 152(2), 439-466 (1999)

3. Hiroshi, M.: Convergence of solutions of one-dimensional semilinear parabolic equations. J. Math. Kyoto Univ. 18(2), 221-227 (1978)

4. Donald, A., Michael, C.: Peletier Lambertus Adrianus., Stabilization of solutions of a degenerate nonlinear diffusion problem. Nonlinear Anal. 6(10), 1001-1022 (1982) 
5. Flavia, S., Tesei, A.: Degenerate regularization of forward-backward parabolic equations: the regularized problem. Arch. Ration. Mech. Anal. 204(1), 85-139 (2012)

6. Martinez, P., Vancostenoble, J.: Carleman estimates for one-dimensional degenerate heat equations. J. Evol. Equ. 6(2), 325-362 (2006)

7. Porzio, Michaela, M., Flavia, S., Tesei, A.: Radon measure-valued solutions for a class of quasilinear parabolic equations. Arch. Ration. Mech. Anal. 210(3), 713-772 (2013)

8. Porzio, Michaela, M., Flavia, S., Tesei, A.: Radon measure-valued solutions of nonlinear strongly degenerate parabolic equations. Calc. Var. Partial Differential Equations 51(1-2), $401-437$ (2014)

9. Craig, E.L., Gariepy, R.F.: Measure Theory and Fine Properties of Functions. Studies in Advanced Mathematics. CRC Press, Boca Raton (1992)

10. Mariano, G., Giuseppe, M., Jiří, S.: Cartesian Currents in the Calculus of Variations. I. Cartesian Currents. Springer, Berlin (1998)

11. Knerr Barry, F.: The porous medium equation in one dimension. Trans. Amer. Math. Soc. 234(2), 381-415 (1977)

12. Lions, J.-L.: Quelques Méthodes de Résolution des Problèmes aux Limites Non linéaires (French). Dunod, Gauthier-Villars, Paris (1969)

13. Vázquez, J.: Luis The Porous Medium Equation. Mathematical theory. Oxford Mathematical Monographs. Oxford (2007)

14. Michiel, B., Flavia Terracina Andrea, S., Alberto, T.: Radon measure-valued solutions of first order scalar conservation laws. Adv. Nonlinear Anal. 9(1), 65-107 (2020)

15. Valadier, M.: Young measure. In: Cellina, A. (ed.) Methods of nonconvex analysis in. Lecture notes in Math., vol. 1446, pp. 152-188. Springer, Berlin (1990)

16. Dolzmann, G., Norbert, H., Stefan, M.: Nonlinear elliptic systems with measure-valued right hand side. Math. Z. 226, 545-574 (1997)

17. Nkombo, Q., Li, F., Tathy, C.: Stability properties of Radon measure-valued solutions for a class of nonlinear parabolic equations under Neumann boundary conditions. AIMS Math. 6(11), 12182-12224 (2021)

18. Maria Michaela, P., Flavia, S.: Radon measure-valued solutions for some quasilinear degenerate elliptic equations. Ann. Mat. Pura Appl., 495-532 (194(2)) 2015

19. Seizô, l.: Fundamental solutions of parabolic differential equations and boundary value problems. Jpn. J. Math. 27, 55-102 (1957)

20. Seizô, l.: A boundary value problem of partial differential equations of parabolic type. Duke Math. J. 24, 299-312 (1957)

21. Aleksandrovna, L.O., Alekseevich, S.V., Nikolaevna, U.N.: Linear and Quasilinear Equations of Parabolic Type. Translations of Math. Monographs. Am. Math. Soc., Providence, R. I. (1968)

22. Andrea, D., Daniela, G., Chiara Segura de León Sergio, L.: Quasi-linear parabolic equations with degenerate coercivity having a quadratic gradient term. Ann. Inst. H. Poincaré Anal. Non Linéaire 23(1), 97-126 (2006)

23. Boccardo, L., Maria Michaela, P.: Bounded Solutions for a Class of Quasi-linear Parabolic Problems with a Quadratic Gradient Term, Evolution equations. In: semigroups and functional analysis, Milano, 2000. Progr. Nonlinear Differential Equations Appl., vol. 50, pp. 39-48. Birkhäuser, Basel (2002)

24. Daniele, A., Emmanuele, D.: On the Cauchy problem and initial traces for a class of evolution equations with strongly sources. Ann. Scuola Norm. Sup. PisaCl. Sci. 18(3), 363-441 (1991)

25. Smoller, J.: Shock Waves and Reaction-Diffusion Equations, 2nd edn. Springer, New-York (1994)

26. Luigi, O., Maria Michaela, P., Flavia, S.: Measure-valued solutions of nonlinear parabolic equations with logarithmic diffusion. J. Evol. Equ. 15(3), 609-645 (2015)

27. Jacques, S.: Compact sets in the space $L^{P}(0, T ; B)$. Ann. Mat. Pura Appl. 146, 65-96 (1987)

28. Flavia, S., Tesei, A.: Degenerate regularization of forward-backward parabolic equations: the vanishing viscosity limit. Math. Ann. 355(2), 551-584 (2013)

29. Ildefonso, D.J., Robert, K.: On a nonlinear degenerate parabolic equation in infiltration or evaporation through a porous medium. J. Differential Equations 69(3), 368-403 (1987)

30. Dang Duc, T., Pham Hoang, Q.: Khanh Tran Vu and Tuan Nguyen Huy. Nonlinear case of the 1-D backward heat problem: regularization and error estimate. Z. Anal. Anwend. 26(2), 231-245 (2007)

31. Corrado, M., Alessio, P., Terracina, A.: Qualitative behaviour for one-dimensional strongly degenerate parabolic problems. Interfaces Free Bound. 8(3), 263-280 (2006)

32. Bertsch, M., de Mottoni, P., Peletier, L.A.: Degenerate diffusion and the Stefan problem. Nonlinear Anal. 8(11), $1311-1336(1984)$

33. Galaktionov Victor, A., Luis, V.J.: Geometrical properties of the solutions of one-dimensional nonlinear parabolic equations. Math. Ann. 303(4), 741-769 (1995)

34. Oleěnik, A.O., Kalashnikov, A.S., Yuř-lin', Č.: The Cauchy problem and boundary problems for equations of the type of non-stationary filtration (Russian). Izv. Akad. Nauk SSSR. Ser. Mat. 22, 667-704 (1958)

35. Kamin, S.: Some estimates for solution of the Cauchy problem for equations of a nonstationary filtration. J. Differential Equations 20(2), 321-335 (1976)

36. Kamin, S.: Source-type solutions for equations of nonstationary filtration. J. Math. Anal. Appl. 64(2), 263-276 (1978)

37. Kamin, S.: Similar solutions and the asymptotics of filtration equations. Arch. Rational Mech. Anal. 60(2), 171-183 (1975)

38. Mascia Corrado, Andrea, T., Tesei, A.: Two-phase entropy solutions of a forward-backward parabolic equation. Arch. Ration. Mech. Anal., 887-925 (194(3)) 2009

39. Gilding Brian, H.: A nonlinear degenerate parabolic equation. Ann. Scuola Norm. Sup. Pisa Cl. Sci. 4(3), 393-432 (1977)

40. Gilding Brian, H.: Improved theory for a nonlinear degenerate parabolic equation. Ann. Scuola Norm. Sup. Pisa Cl. Sci. 16(2), 165-224 (1989)

41. Plotnikov Pavel, l.: Passing to the limit with respect to viscosity in an equation with variable parabolicity direction Differ. Equ. 30, 614-622 (1994)

42. Flavia, S.: On a class of equations with variable parabolicity direction. Discrete Contin. Dyn. Syst. 22(3), 729-758 (2008)

43. Luigi, A., Nicola, F., Diego, P.: Functions of Bounded Variation and Free Discontinuity Problems. Oxford Mathematical Monographs. The Clarendon Press, Oxford University Press, New York (2000) 
44. Silvia Van der Mee Cornelis, F., Giuseppe, V.: Boundedness in a nonlinear attraction-repulsion Keller-Segel system with production and consumption. J. Math. Anal. Appl. 504(2), Article ID 125428 (2021)

45. Tongxing, L., Nicola, P., Giuseppe, V.: Properties of solutions to porous medium problems with different sources and boundary conditions. Z. Angew. Math. Phys. 70(3), Article ID 86 (2019)

46. Tongxing, L., Giuseppe, V.: Boundedness for a nonlocal reaction chemotaxis model even in the attraction-dominated regime. Differential Integral Equations 34(5-6), 315-336 (2021)

Submit your manuscript to a SpringerOpen ${ }^{\odot}$ journal and benefit from:

- Convenient online submission

$\checkmark$ Rigorous peer review

- Open access: articles freely available online

- High visibility within the field

- Retaining the copyright to your article

Submit your next manuscript at $\gg$ springeropen.com 